\title{
Recent progress in elliptic equations and systems of arbitrary order with rough coefficients in Lipschitz domains
}

\author{
Vladimir Maz'ya • Tatyana Shaposhnikova
}

Received: 7 September 2010 / Accepted: 27 January 2011 / Published online: 9 March 2011

(C) The Author(s) 2011. This article is published with open access at SpringerLink.com

\begin{abstract}
This is a survey of results mostly relating elliptic equations and systems of arbitrary even order with rough coefficients in Lipschitz graph domains. Asymptotic properties of solutions at a point of a Lipschitz boundary are also discussed.
\end{abstract}

Keywords Higher order elliptic equations - Higher order elliptic systems · Besov spaces · BMO - VMO - Lipschitz graph domains · Green's function · Asymptotic behaviour of solutions

Mathematics Subject Classification (2010) Primary 35G15 · 35J55;

Secondary $35 \mathrm{~J} 67 \cdot 35 \mathrm{E} 05$

\section{Introduction}

Results on the regularity of solutions up to the boundary of a domain play a very important role in the theory of linear elliptic equations and systems. The following classical example serves as an illustration.

Communicated by A. Laptev.

V. Maz’ya

Department of Mathematical Sciences, University of Liverpool,

M\&O Building, Liverpool L69 3BX, UK

e-mail: vladimir.mazya@liu.se

V. Maz’ya · T. Shaposhnikova $(\varangle)$

Department of Mathematics, Linköping University, 58183 Linköping, Sweden

e-mail: tatiana.shaposhnikova@liu.se 
Consider the Dirichlet problem

$$
\begin{cases}\Delta u=0 & \text { in } \quad \Omega, \\ \operatorname{Tr} u=f & \text { on } \quad \partial \Omega\end{cases}
$$

where $\Omega$ is a bounded domain with smooth boundary in $\mathbb{R}^{n}$ and $\operatorname{Tr} u$ stands for the boundary value (trace) of $u$. Let $u \in W_{p}^{1}(\Omega), 1<p<\infty$, that is

$$
\|u\|_{W_{p}^{1}(\Omega)}:=\left(\int_{\Omega}\left(|\nabla u|^{p}+|u|^{p}\right) d x\right)^{1 / p}<\infty,
$$

and let $f$ belong to the Besov space $B_{p}^{1-1 / p}(\partial \Omega)$ with the norm

$$
\left(\int_{\partial \Omega} \int_{\partial \Omega} \frac{|f(x)-f(y)|^{p}}{|x-y|^{n+p-2}} d \sigma_{x} d \sigma_{y}+\int_{\partial \Omega}|f(x)|^{p} d \sigma_{x}\right)^{1 / p} .
$$

It is well-known that

$$
\operatorname{Tr} W_{p}^{1}(\Omega)=B_{p}^{1-1 / p}(\partial \Omega)
$$

Moreover, the harmonic extension $u$ of $f \in B_{p}^{1-1 / p}(\partial \Omega)$ belongs to $W_{p}^{1}(\Omega)$ and the norm of $u$ in $W_{p}^{1}(\Omega)$ is equivalent to the above norm in $B_{p}^{1-1 / p}(\partial \Omega)$.

This fact might be the starting point for the following topics of interest:

- Consider the Dirichlet problem for a general elliptic operator with variable coefficients and study the impact of low regularity assumptions for the coefficients on solvability in Sobolev spaces.

- Instead of the domain with smooth boundary consider a domain in a more general class and study the effect of irregularities of $\partial \Omega$ on solvability properties of boundary value problems.

- Understand the relation between the smoothness of boundary data and the smoothness of solutions.

Another important theme, somehow related to these, is:

- Describe the local behaviour of solutions near a boundary point or interior point of the domain $\Omega$ under weak assumptions on the coefficients and the geometry of the domain.

In what follows we survey results in the directions just listed which concern elliptic equations and systems of arbitrary even order with rough coefficients in Lipschitz graph domains. Most of these results were obtained by ourselves and our collaborators during the last decade. The papers specific for differential operators of the second order are usually only mentioned. 
Here is the plan of the article. Section 1 is dedicated to weak solutions of the Dirichlet problem with Besov boundary data, with the coefficients of the differential operators and the unit normal to the boundary with small local mean oscillation. Results of a similar nature for the Stokes system are discussed in Sect. 2. In Sect. 3, strong solutions in Sobolev spaces are considered. Here sharp additional conditions on the Lipschitz boundary are reviewed. In particular, in Sect. 3.2 we speak about strong solvability of the Stokes system. Section 4 concerns asymptotic formulas for solutions near an isolated point of the Lipschitz boundary and at a point in the domain.

\section{Weak solvability of the Dirichlet problem for higher order elliptic systems in Lipschitz domains with boundary data in Besov classes}

\subsection{Background}

The present section is mostly based on results of the paper by Maz'ya et al. [51,52]. We start by mentioning earlier works.

The basic case of the Laplacian in arbitrary Lipschitz graph domains in $\mathbb{R}^{n}$, is treated in the work of Dahlberg and Kenig [18], in the case of $L_{p}$-data, and Jerison and Kenig [36], in the case of data in the Besov space $B_{p}^{s}$ with $0<s<1$. The local regularity in the Sobolev class $W_{p}^{2}$ of solutions to second order equations with coefficients in $V M O \cap L_{\infty}$ was established by Chiarenza et al. [15].

In spite of substantial progress in recent years, there remain many basic open questions for higher order equations, even in the case of constant coefficient operators in Lipschitz domains. Recall that a domain $\Omega$ is called Lipschitz graph if its boundary can be locally described by means of (appropriately rotated and translated) graphs of realvalued Lipschitz functions. One significant problem is to determine the sharp range of $p$ 's for which the Dirichlet problem for strongly elliptic systems with $L_{p}$-boundary data is well-posed. Pipher and Verchota [63] have developed a $L_{p}$-theory for real, constant coefficient, higher order systems

$$
L=\sum_{|\alpha|=2 m} A_{\alpha} D^{\alpha}
$$

when $p$ is near 2, i.e., $2-\varepsilon<p<2+\varepsilon$ with $\varepsilon>0$ depending on the Lipschitz character of $\Omega$. On p. 2 of [63] the authors ask whether the $L_{p}$-Dirichlet problem for these operators is solvable in a given Lipschitz domain for $p \in\left(2-\varepsilon, \frac{2(n-1)}{n-3}+\varepsilon\right)$, and a positive answer has been given by Shen [66]. Let us also mention the work [2] of Adolfsson and Pipher on the Dirichlet problem for the biharmonic operator in arbitrary Lipschitz domains and with data in Besov spaces, as well as [71] where Verchota formulates and solves a Neumann-type problem for the bi-Laplacian in Lipschitz domains and with boundary data in $L_{2}$. Mitrea et al. [59] treat the Dirichlet problem for strongly elliptic systems of second order in an arbitrary Lipschitz subdomain $\Omega$ of a (smooth) Riemannian manifold and with boundary data in $B_{p}^{s}(\partial \Omega)$, when $2-\varepsilon<p<2+\varepsilon$ and $0<s<1$. 
We mention some recent results on the second order elliptic equations and systems with coefficients in VMO due to Di Fazio [23], Caffarelli and Peral [12], Stroffolini [68], Guidetti [32], Auscher and Qafsaouti [9], for higher order equations see also Palagachev and Softova [62]. Recently Dindoš et al. obtained interesting facts concerning the solvability of the Dirichlet problem with $B M O$ boundary data for second order scalar uniformly elliptic equations with real coefficients on a very general class of domains, including Lipschitz and polyhedral domains [24]. Deep results in the $L_{2}$-theory of the Dirichlet problem for second order uniformly elliptic equations were achieved by Aucher, Axelsson, Hofman, Lacey, McIntosh, Tchamitchian et al., which are connected, in particular, with Kato's square root problem, see [6-8] and references there.

\subsection{Domains and function spaces}

Let us turn to the article [51]. We make no notational distinction between spaces of scalar-valued functions and their natural counterparts for vector-valued functions.

It has been shown by Hofmann et al. [34] that $\Omega$ is a Lipschitz graph domain if and only if it has finite perimeter in the sense of De Giorgi (see $[11,21,28]$ ) and (i) there are continuous (or, equivalently, smooth) vector fields that are transversal to the boundary and (ii) the necessary condition $\partial \Omega=\partial \bar{\Omega}$ is fulfilled. An equivalent characterization obtained in [34] is as follows. A bounded nonempty domain of finite perimeter for which $\partial \Omega=\partial \bar{\Omega}$ is a Lipschitz graph domain if and only if

$$
\inf \left\{\|v-\omega\|_{L_{\infty}(\partial \Omega)}: \omega=\left(\omega_{1}, \ldots, \omega_{n}\right) \in C(\partial \Omega),|\omega|=1 \text { on } \partial \Omega\right\}<\sqrt{2}
$$

with $v$ being the outward normal to $\partial \Omega$.

Everywhere in this section we assume that $\Omega$ is a Lipschitz graph domain in $\mathbb{R}^{n}$, with compact closure $\bar{\Omega}$ and with the outward unit normal $v=\left(v_{1}, \ldots, v_{n}\right)$. Let $m$ be an integer. Consider the operator

$$
\mathcal{L}\left(X, D_{X}\right) \mathcal{U}:=\sum_{|\alpha|=|\beta|=m} D^{\alpha}\left(A_{\alpha \beta}(X) D^{\beta} \mathcal{U}\right), \quad X \in \Omega
$$

with the data

$$
\frac{\partial^{k} \mathcal{U}}{\partial \nu^{k}}=g_{k} \quad \text { on } \partial \Omega, \quad 0 \leq k \leq m-1
$$

The coefficients $A_{\alpha \beta}$ are square matrices with measurable, complex-valued entries, for which there exists $\kappa>0$ such that

$$
\sum_{|\alpha|=|\beta|=m}\left\|A_{\alpha \beta}\right\|_{L_{\infty}(\Omega)} \leq \kappa^{-1}
$$


and the coercivity condition

$$
\operatorname{Re} \int_{\Omega} \sum_{|\alpha|=|\beta|=m}\left\langle A_{\alpha \beta}(X) D^{\beta} V(X), D^{\alpha} V(X)\right\rangle d X \geq \kappa \sum_{|\alpha|=m}\left\|D^{\alpha} V\right\|_{L_{2}(\Omega)}^{2}
$$

holds for all complex vector-valued functions $V \in C_{0}^{\infty}(\Omega)$. By the Lax-Milgram lemma, this condition guarantees the unique solvability in the variational sense of the Dirichlet problem for the operator $\mathcal{L}$ with zero boundary conditions.

Let $\mathcal{U}$ belong to the usual Sobolev space $W_{p}^{m}(\Omega)$. It is natural to take

$$
\frac{\partial^{k} \mathcal{U}}{\partial v^{k}}:=\sum_{|\alpha|=k} \frac{k !}{\alpha !} v^{\alpha} \operatorname{Tr}\left[D^{\alpha} \mathcal{U}\right], \quad 0 \leq k \leq m-1
$$

where $v^{\alpha}:=v_{1}^{\alpha_{1}}, \ldots, v_{n}^{\alpha_{n}}$ if $\alpha=\left(\alpha_{1}, \ldots, \alpha_{n}\right)$. Now, let $p \in(1, \infty), a \in(-1 / p, 1-$ $1 / p$ ) be fixed and let

$$
\rho(X):=\operatorname{dist}(X, \partial \Omega) \text {. }
$$

The space $W_{p}^{m, a}(\Omega)$ is defined as the space of vector-valued functions for which

$$
\sum_{|\alpha| \leq m} \int_{\Omega}\left|D^{\alpha} \mathcal{U}(X)\right|^{p} \rho(X)^{p a} d X<\infty
$$

Needless to say, $W_{p}^{m, a}(\Omega)$ is the Sobolev space $W_{p}^{m}(\Omega)$ when $a=0$. Further we let $\stackrel{\circ}{W}_{p}^{m, a}(\Omega)$ be the closure of $C_{0}^{\infty}(\Omega)$ in $W_{p}^{m, a}(\Omega)$. The dual space is given by

$$
W_{p^{\prime}}^{-m,-a}(\Omega):=\left(\stackrel{\circ}{W}_{p}^{m, a}(\Omega)\right)^{*}
$$

(In the case $a=0$ one uses the alternative notations $\stackrel{\circ}{W}_{p}^{m}(\Omega)$ and $W_{p^{\prime}}^{-m}(\Omega)$.)

For any $\mathcal{U} \in W_{p}^{m, a}(\Omega)$ the traces of $D^{\alpha} \mathcal{U}, 0 \leq|\alpha| \leq m-1$, exist in $B_{p}^{s}(\partial \Omega)$, where $s:=1-a-1 / p, 0<s<1$ (see [29] for $a=0$ and [70]). Recall that $f \in L_{p}(\partial \Omega$ ) belongs to $B_{p}^{s}(\partial \Omega)$ if and only if

$$
\int_{\partial \Omega} \int_{\partial \Omega} \frac{|f(X)-f(Y)|^{p}}{|X-Y|^{n-1+s p}} d \sigma_{X} d \sigma_{Y}<\infty
$$

The above definition takes advantage of the Lipschitz manifold structure of $\partial \Omega$ which allows one to define smoothness spaces of index less than 1 . This approach is no longer effective when the order of smoothness exceeds 1 .

Let us illustrate the necessity of working with boundary data different from those in spaces of traces of usual Sobolev spaces by considering the Dirichlet problem for 
the biharmonic operator

$$
\begin{aligned}
& \mathcal{U} \in W_{2}^{2}(\Omega), \quad \Delta^{2} \mathcal{U}=0 \text { in } \Omega, \\
& \operatorname{Tr} \mathcal{U}=g_{0} \quad \text { on } \partial \Omega, \quad\langle\nu, \operatorname{Tr}[\nabla \mathcal{U}]\rangle=g_{1} \quad \text { on } \partial \Omega .
\end{aligned}
$$

One might be tempted to believe that a natural class of boundary data is $B_{2}^{3 / 2}(\partial \Omega) \times$ $B_{2}^{1 / 2}(\partial \Omega)$, where by definition $B_{2}^{3 / 2}(\partial \Omega)$ and $B_{2}^{1 / 2}(\partial \Omega)$ are the spaces of traces of functions in $W_{2}^{2}(\Omega)$ and $W_{2}^{1}(\Omega)$, respectively.

However, this formulation has serious drawbacks. The first one is that the mapping

$$
W_{2}^{2}(\Omega) \ni \mathcal{U} \mapsto\langle v, \operatorname{Tr}[\nabla \mathcal{U}]\rangle \in B_{2}^{1 / 2}(\partial \Omega)
$$

is generally unbounded. Setting $\mathcal{U}=x_{i}$, we see that the continuity of this mapping would imply $v \in B_{2}^{1 / 2}(\partial \Omega)$. This fails even for the square $S=[0,1]^{2}$.

Secondly, the problem (1.6), (1.7) is not always solvable when $\left(g_{0}, g_{1}\right)$ is an arbitrary pair in $B_{2}^{3 / 2}(\partial \Omega) \times B_{2}^{1 / 2}(\partial \Omega)$. Indeed, consider the case $\Omega=S$ and $g_{0}=0$, $g_{1}=1$. It is known and follows from Kondratiev's theory [40] that the main term of the asymptotics near the origin of any solution $\mathcal{U}$ in $W_{2}^{2}(S)$ is given in polar coordinates $(r, \theta)$ by

$$
\frac{2 r}{\pi+2}\left(\left(\theta-\frac{\pi}{2}\right) \sin \theta-\theta \cos \theta\right)
$$

Since this function does not belong to $W_{2}^{2}(S)$, there is no solution in this space.

A new point of view has been introduced by Whitney [72] who considered higher order Lipschitz spaces on arbitrary closed sets. An extension of this circle of ideas pertaining to the full scale of Besov and Sobolev spaces on irregular subsets of $\mathbb{R}^{n}$ can be found in the book by Jonsson and Wallin [38]. The authors of [51] further refined this theory in the context of Lipschitz domains. The description of higher order Besov spaces on the boundary of a Lipschitz domain $\Omega \subset \mathbb{R}^{n}$ in [51] runs as follows.

For $m \in \mathbb{N}, p \in(1, \infty), s \in(0,1)$, the space $\dot{B}_{p}^{m-1+s}(\partial \Omega)$ is introduced as the closure of

$$
\left\{\left(\left.D^{\alpha} \mathcal{V}\right|_{\partial \Omega}\right)_{|\alpha| \leq m-1}: \mathcal{V} \in C_{0}^{\infty}\left(\mathbb{R}^{n}\right)\right\}
$$

in $B_{p}^{s}(\partial \Omega)$. An equivalent characterization of $\dot{B}_{p}^{m-1+s}(\partial \Omega)$ which involves higher order Taylor remainder in place of $f(X)-f(Y)$ in (1.5) runs as follows (see Sect. 7.1 of [51]). 
For a collection of families $\dot{f}=\left\{f_{\alpha}\right\}_{|\alpha| \leq m-1}$ of measurable functions defined on $\partial \Omega$, there is the equivalence of norms

$$
\begin{aligned}
\|\dot{f}\|_{\dot{B}_{p}^{m-1+s}(\partial \Omega)} \sim & \sum_{|\alpha| \leq m-1}\left\|f_{\alpha}\right\|_{L_{p}(\partial \Omega)} \\
& +\sum_{|\alpha| \leq m-1}\left(\int_{\partial \Omega} \int_{\partial \Omega} \frac{\left|R_{\alpha}(X, Y)\right|^{p}}{|X-Y|^{p(m-1+s-|\alpha|)+n-1}} d \sigma_{X} d \sigma_{Y}\right)^{1 / p}
\end{aligned}
$$

where

$$
R_{\alpha}(X, Y):=f_{\alpha}(X)-\sum_{|\beta| \leq m-1-|\alpha|} \frac{1}{\beta !} f_{\alpha+\beta}(Y)(X-Y)^{\beta}, \quad X, Y \in \partial \Omega
$$

Here and elsewhere by the equivalence $a \sim b$ we mean that $c_{1} a \leq b \leq c_{2} a$ with positive constants $c_{1}, c_{2}$ depending on $n, m, p, s$ and similar parameters.

It is easy to prove that $\dot{B}_{p}^{m-1+s}(\partial \Omega)$ is a Banach space. Also, trivially, for any constant $\kappa>0$,

$\sum_{|\alpha| \leq m-1}\left\|f_{\alpha}\right\|_{L_{p}(\partial \Omega)}+\sum_{|\alpha| \leq m-1}\left(\int_{\substack{X, Y \in \partial \Omega \\|X-Y|<\kappa}} \frac{\left|R_{\alpha}(X, Y)\right|^{p}}{|X-Y|^{p(m-1+s-|\alpha|)+n-1}} d \sigma_{X} d \sigma_{Y}\right)^{1 / p}$

is an equivalent norm on $\dot{B}_{p}^{m-1+s}(\partial \Omega)$.

In order to formulate the trace and extension theorem for the spaces $\dot{B}_{p}^{m-1+s}(\partial \Omega)$, we first give its analogue for lower smoothness spaces which is essentially due to [70].

Lemma 1 For each $1<p<\infty,-1 / p<a<1-1 / p$ and $s:=1-a-1 / p$, the trace operator

$$
\operatorname{Tr}: W_{p}^{1, a}(\Omega) \longrightarrow B_{p}^{s}(\partial \Omega)
$$

is well-defined, linear, bounded, onto and has $\stackrel{\circ}{W}_{p}^{1, a}(\Omega)$ as its null-space. Furthermore, there exists a linear, continuous mapping

$$
\mathcal{E}: B_{p}^{s}(\partial \Omega) \longrightarrow W_{p}^{1, a}(\Omega)
$$

called extension operator, such that $\operatorname{Tr} \circ \mathcal{E}=I$ (i.e., a bounded, linear right-inverse of trace).

For higher smoothness see the following assertion which is Proposition 7.3 in [51]. 
Proposition 1 For $1<p<\infty,-1 / p<a<1-1 / p, s:=1-a-1 / p \in(0,1)$ and $m \in \mathbb{N}$, define the higher order trace operator

$$
t r_{m-1}: W_{p}^{m, a}(\Omega) \longrightarrow \dot{B}_{p}^{m-1+s}(\partial \Omega)
$$

by setting

$$
\operatorname{tr}_{m-1} \mathcal{U}:=\left\{i^{|\alpha|} \operatorname{Tr}\left[D^{\alpha} \mathcal{U}\right]\right\}_{|\alpha| \leq m-1},
$$

where the traces in the right-hand side are taken in the sense of Lemma 1. Then (1.12) is a well-defined, linear, bounded operator, which is onto and has $\stackrel{\circ}{W}_{p}^{m, a}(\Omega)$ as its null-space. Moreover, it has a bounded, linear right-inverse, i.e., there exists a linear, continuous operator

$$
\mathcal{E}: \dot{B}_{p}^{m-1+s}(\partial \Omega) \longrightarrow W_{p}^{m, a}(\Omega)
$$

such that

$$
\dot{f}=\left\{f_{\alpha}\right\}_{|\alpha| \leq m-1} \in \dot{B}_{p}^{m-1+s}(\partial \Omega) \text { implies } \operatorname{tr}_{m-1}(\mathcal{E} \dot{f})=f_{\alpha},
$$

i.e. $t r_{m-1} \circ \mathcal{E}=I$.

Now, the boundary data $\left\{g_{k}\right\}_{0 \leq k \leq m-1}$ in the Dirichlet problem (1.2) are understood in the following sense:

$$
\begin{aligned}
& \text { there exists an array } \dot{f}=\left\{f_{\alpha}\right\}_{|\alpha| \leq m-1} \in \dot{B}_{p}^{m-1+s}(\partial \Omega) \text { such that } \\
& g_{k}=\sum_{|\alpha|=k} \frac{k !}{\alpha !} v^{\alpha} f_{\alpha} \quad \text { for each } 0 \leq k \leq m-1
\end{aligned}
$$

The set of all families of $\left\{g_{k}\right\}$ is a Banach space denoted by $\dot{W}_{p}^{m-1+s}(\partial \Omega)$.

The space takes a particularly simple form when $m=2$. To describe it, we need the notation for the tangential derivative $\partial / \partial \tau_{j k}$ given by

$$
\frac{\partial}{\partial \tau_{j k}}:=v_{j} \frac{\partial}{\partial x_{k}}-v_{k} \frac{\partial}{\partial x_{j}}, \quad 1 \leq j, k \leq n,
$$

and the tangential gradient on the surface $\partial \Omega$

$$
\nabla_{\tan }:=\left(\sum_{j} v_{j} \partial / \partial \tau_{j k}\right)_{1 \leq k \leq n} .
$$

Then, for each Lipschitz graph domain $\Omega \subset \mathbb{R}^{n}$ and each $1<p<\infty, s \in(0,1)$,

$$
\dot{W}_{p}^{1+s}(\partial \Omega)=\left\{\left(g_{0}, g_{1}\right) \in L_{p}^{1}(\partial \Omega) \oplus L_{p}(\partial \Omega): v g_{1}+\nabla_{\tan } g_{0} \in B_{p}^{s}(\partial \Omega)\right\} \text {. }
$$


This has been conjectured to hold (when $s=1-1 / p$ ) by Buffa and Geymonat on p. 703 of [10] and proved in [51], Corollary 7.11.

1.3 Solvability of the Dirichlet problem in $W_{p}^{m, a}(\Omega)$

Consider the Dirichlet problem for the operator (1.1)

$$
\begin{cases}\mathcal{L}\left(X, D_{X}\right) \mathcal{U}=\mathcal{F} & \text { in } \Omega \\ \frac{\partial^{k} \mathcal{U}}{\partial v^{k}}=g_{k} & \text { on } \partial \Omega, 0 \leq k \leq m-1\end{cases}
$$

The following assertion is proved in [51].

Proposition 2 If $\mathcal{U} \in W_{p}^{m, a}(\Omega)$ then, necessarily,

$$
\mathcal{F} \in W_{p}^{-m, a}(\Omega), g:=\left\{g_{k}\right\}_{0 \leq k \leq m-1} \in \dot{W}_{p}^{m-1+s}(\partial \Omega)
$$

and, moreover,

$$
\|g\|_{\dot{W}_{p}^{m-1+s}(\partial \Omega)}+\|\mathcal{F}\|_{W_{p}^{-m, a}(\Omega)} \leq C\|\mathcal{U}\|_{W_{p}^{m, a}(\Omega)} \cdot
$$

Results in the converse direction were also established in [51]. The main hypothesis in [51] requires that, at small scales, the so called local mean oscillations of the unit normal to $\partial \Omega$ and of the coefficients of the differential operator $\mathcal{L}\left(X, D_{X}\right)$ are not too large, relative to the Lipschitz constant of the domain $\Omega$, the ellipticity constant of $\mathcal{L}\left(X, D_{X}\right)$, and the indices of the corresponding Besov space.

We use the following notation for the mean value of a function $g$ on a measurable set $E$ :

$$
f_{E} g(x) d x=\frac{1}{|E|} \int_{E} g(x) d x
$$

with $|E|$ standing for the Lebesgue measure of the set $E$.

By the local mean oscillation of $F \in L_{1}(\Omega)$ we understand

$$
\{F\}_{\operatorname{Osc}(\Omega)}:=\lim _{\varepsilon \rightarrow 0}\left(\sup _{\left\{B_{\varepsilon}\right\}_{\Omega}} \underset{B_{\varepsilon} \cap \Omega}{f} \underset{B_{\varepsilon} \cap \Omega}{f}|F(x)-F(y)| d x d y\right),
$$

where $\left\{B_{\varepsilon}\right\}_{\Omega}$ stands for the family of balls of radius $\varepsilon$ centered at points of $\Omega$. Similarly, the local mean oscillation of $f \in L_{1}(\partial \Omega)$ is

$$
\{f\}_{\operatorname{Osc}(\partial \Omega)}:=\lim _{\varepsilon \rightarrow 0}\left(\sup _{\left\{B_{\varepsilon}\right\}_{\partial \Omega}} f f_{B_{\varepsilon} \cap \partial \Omega} f|f(x)-f(y)| d s_{x} d s_{y} \cap\right),
$$


where $\left\{B_{\varepsilon}\right\}_{\partial \Omega}$ is the collection of $n$-dimensional balls of radius $\varepsilon$ with centers on $\partial \Omega$.

We recall that a locally integrable function $g$ in $\mathbb{R}^{n}$ belongs to the space $B M O\left(\mathbb{R}^{n}\right)$ if

$$
\|g\|_{B M O\left(\mathbb{R}^{n}\right)}:=\sup _{B} f_{B}\left|g(x)-f_{B} g(y) d y\right| d x
$$

is finite, where the supremum is taken over all balls $B$ in $\mathbb{R}^{n}$. The above supremum defines a seminorm in $B M O\left(\mathbb{R}^{n}\right)$.

Note that smallness of the local mean oscillation of the normal does not imply smallness of the Lipschitz constant of $\partial \Omega$. Indeed, let

$$
\Omega=\left\{(x, y) \in \mathbb{R}^{2}, y>\varphi_{\varepsilon}(x)\right\},
$$

where

$$
\varphi_{\varepsilon}(x)=x \sin \left(\varepsilon \log |x|^{-1}\right)
$$

Then $\left\|\varphi_{\varepsilon}^{\prime}\right\|_{L_{\infty}(\mathbb{R})} \sim 1$, while $\left\|\varphi_{\varepsilon}^{\prime}\right\|_{B M O(\mathbb{R})} \leq C \varepsilon$.

Let $\Omega \subset \mathbb{R}^{n}$ be a bounded Lipschitz domain whose Lipschitz constant does not exceed $M$, and assume that the operator $\mathcal{L}\left(X, D_{X}\right)$ of order $2 m$ is strongly elliptic and has bounded, measurable complex coefficients. Consider the Dirichlet problem

$$
\left\{\begin{array}{l}
\sum_{|\alpha|=|\beta|=m} D^{\alpha}\left(A_{\alpha \beta}(X) D^{\beta} \mathcal{U}\right)=\mathcal{F} \text { for } X \in \Omega, \\
\frac{\partial^{k} \mathcal{U}}{\partial \nu^{k}}=g_{k} \quad \text { on } \partial \Omega, \quad 0 \leq k \leq m-1,
\end{array}\right.
$$

with $\mathcal{F} \in W_{p}^{-m, a}(\Omega)$ and $g:=\left\{g_{k}\right\}_{0 \leq k \leq m-1}$ in $\dot{W}_{p}^{m-1+s}(\partial \Omega)$.

The main result in [51] runs as follows.

Theorem 1 There exists a positive constant $C$, depending only on $M$ and the ellipticity constant of $\mathcal{L}$, such that: For each $p \in(1, \infty), s \in(0,1)$ and $a:=1-s-1 / p$, the problem (1.19) has a unique solution $\mathcal{U} \in W_{p}^{m, a}(\Omega)$ if the coefficient matrices $A_{\alpha \beta}$ and the exterior normal vector $v$ to $\partial \Omega$ satisfy

$$
\begin{aligned}
& \{v\}_{\operatorname{Osc}(\partial \Omega)}+\sum_{|\alpha|=|\beta|=m}\left\{A_{\alpha \beta}\right\}_{O S c(\Omega)} \\
& \leq C s(1-s)\left(p p^{\prime}+s^{-1}(1-s)^{-1}\right)^{-1} .
\end{aligned}
$$

For second order operators the factor $s(1-s)$ in the last inequality can be removed. Furthermore, there exists $C=C\left(\partial \Omega, A_{\alpha \beta}, p, s\right)>0$ such that

$$
\|\mathcal{U}\|_{W_{p}^{m, a}(\Omega)} \leq C\left(\|g\|_{\dot{W}_{p}^{m-1+s}(\partial \Omega)}+\|\mathcal{F}\|_{W_{p}^{-m, a}(\Omega)}\right)
$$


Let $B M O$ and $V M O$ stand, respectively, for the space of functions of bounded mean oscillations and the space of functions of vanishing mean oscillations (considered either on $\Omega$, or on $\partial \Omega$ ). We recall that the space $V M O$ is defined as the closure in $B M O$ of the space of uniformly continuous functions. It can be proved that

$$
\{F\}_{\text {Osc }} \sim \operatorname{dist}(F, V M O)
$$

where the distance is taken in $B M O$. Thus the small oscillation condition introduced in Theorem 1 holds if

$$
\begin{aligned}
& \operatorname{dist}(v, V M O)+\sum_{|\alpha|=|\beta|=m} \operatorname{dist}\left(A_{\alpha \beta}, V M O\right) \\
& \leq C s(1-s)\left(p p^{\prime}+s^{-1}(1-s)^{-1}\right)^{-1} .
\end{aligned}
$$

This is the case if, e.g., $\|v\|_{B M O}+\sum\left\|A_{\alpha \beta}\right\|_{B M O}$ is sufficiently small hence, trivially, if $\nu \in V M O(\partial \Omega)$ and $A_{\alpha \beta}$ belong to $\operatorname{VMO}(\Omega)$, irrespective of $p, s, \mathcal{L}$ and $\Omega$.

Other examples of domains satisfying the hypotheses of Theorem 1 are: Lipschitz graph domains with a sufficiently small Lipschitz constant, relatively to the exponents $p$ and $s$. In particular, Lipschitz graph polyhedral domains with dihedral angles sufficiently close to $\pi$, depending on $p$ and $s$.

As a further comment to Theorem 1, we note that the innovation in [52] that allows to consider boundary data in higher-order Besov spaces, is the systematic use of weighted Sobolev spaces. In relation to the standard Besov scale, we would like to point out that

$$
W_{p}^{m, a}(\Omega) \hookrightarrow B_{p}^{m-1+s+1 / p}(\Omega) \text { for } a=1-s-\frac{1}{p} \in(0,1-1 / p)
$$

and

$$
B_{p}^{m-1+s+1 / p}(\Omega) \hookrightarrow W_{p}^{m, a}(\Omega) \text { for } a=1-s-\frac{1}{p} \in(-1 / p, 0)
$$

According to Theorem 1, for a Lipschitz $\partial \Omega$, we have

$$
\sum_{|\alpha| \leq m-1}\left\|\operatorname{Tr}\left[D^{\alpha} \mathcal{U}\right]\right\|_{B_{p}^{s}(\partial \Omega)} \sim\left(\sum_{|\alpha| \leq m} \int_{\Omega} \rho(X)^{p(1-s)-1}\left|D^{\alpha} \mathcal{U}(X)\right|^{p} d X\right)^{1 / p}
$$

uniformly in $\mathcal{U}$ satisfying $\mathcal{L}\left(X, D_{X}\right) \mathcal{U}=0$ in $\Omega$.

This generalizes the trace and extension result by Uspenskiı̌ [70] according to which

$$
\|\operatorname{Tr} u\|_{B_{p}^{s}\left(\mathbb{R}^{n-1}\right)} \sim\left(\int_{\mathbb{R}_{+}^{n}} x_{n}^{p(1-s)-1}\left|\nabla u\left(x^{\prime}, x_{n}\right)\right|^{p} d x\right)^{1 / p}
$$


if $1<p<\infty$ and $0<s<1$, for $u$ harmonic in the upper-half space.

Of course, condition (1.3) ensures that the left-hand side of (1.20) is always finite, but it is its actual size which determines whether for a given pair of indices $s, p$, the problem (1.2), (1.4), (1.16) is well-posed. Note that the maximum value that the righthand side of (1.20) takes for $0<s<1$ and $1<p<\infty$ occurs precisely when $p=2$ and $a:=1-s-1 / p=0$. As (1.20) shows, the set of pairs $(s, 1 / p) \in(0,1) \times(0,1)$ for which (1.2) is well-posed in the context of Theorem 1 exhausts the entire square $(0,1) \times(0,1)$ as the distance from $v$ and the $A_{\alpha \beta}$ 's to $V M O$ tends to zero (while the Lipschitz constant of $\Omega$ and the ellipticity constant of $\mathcal{L}$ stay bounded).

The next assertion, obtained in [51], ensures the unique solvability of the problem (1.19) under very general assumptions on $\partial \Omega$ and the coefficients provided that $p$ is close to 2 and $a$ is small.

Theorem 2 Let $\Omega \subset \mathbb{R}^{n}$ be a bounded Lipschitz domain whose Lipschitz constant does not exceed $M$, and assume that the operator $\mathcal{L}\left(X, D_{X}\right)$ of order $2 m$ is strongly elliptic, and has bounded, measurable (complex) coefficients. Then there exists a positive $\varepsilon$, depending only on $M$ and the ellipticity constant of $\mathcal{L}$, such that:

For each $p \in(1, \infty), s \in(0,1)$ and $a:=1-s-1 / p$, the Dirichlet problem for $\mathcal{L}$ with $\mathcal{F} \in W_{p}^{-m, a}(\Omega)$ and $g:=\left\{g_{k}\right\}_{0 \leq k \leq m-1}$ in $\dot{W}_{p}^{m-1+s}(\partial \Omega)$ has a unique solution $\mathcal{U} \in W_{p}^{m, a}(\Omega)$ if

$$
\left|2^{-1}-p^{-1}\right|<\varepsilon \text { and }|a|<\varepsilon,
$$

Recently Agranovich [3] obtained this type of results $(|p-2|$ is small and $0<$ $s<1$ ) for both Dirichlet and Neumann problems for a subclass of strongly elliptic systems with Douglis-Nirenberg structure in bounded Lipschitz domains. Unlike Theorem 2 on regularity in the weighted Sobolev space $W_{p}^{m, a}(\Omega)$, the regularity results in [3] concern solutions in spaces of Bessel potentials $H_{p}^{\sigma}$ and Besov spaces $B_{p}^{\sigma}$ with uniformly Lipschitz coefficients. His approach is based on regularity methods due to Savaré as well as on author's developement of interpolation theory of spaces $H_{p}^{\sigma}$ and $B_{p}^{\sigma}$ with $\sigma$ of arbitrary sign (see [4] for more details), where the essential role is played by an extension operator from $\Omega$ to $\mathbb{R}^{n}$ introduced by Rychkov [65]. To be specific, Agranovich considered a Douglis-Nirenberg system with the principal part $L_{0}$ whose entries are given by

$$
L_{j, k}(x, D)=\sum_{\substack{|\alpha|=m_{j},|\beta|=m_{k}}} D^{\alpha}\left(a_{\alpha, \beta}^{j, k}(x) D^{\beta}\right) .
$$

The coefficients $a_{\alpha, \beta}^{j, k}(x)$ are complex-valued and no formal self-adjointness of the operatot $L_{j, k}$ is assumed. The principal symbol of the system, i.e. the matrix $L_{0}(x, \xi)$ with entries $L_{j, k}(x, \xi)$, is strongly elliptic:

$$
\operatorname{Re} L_{0}(x, \xi) \geq C \Lambda(\xi)
$$


where $\Lambda(\xi)$ is the diagonal matrix with entries $|\xi|^{2 m_{j}}$ on the main diagonal, $j=$ $1, \ldots, l$, and $C$ is a positive constant. The Savaré method requires the additional condition

$$
\operatorname{Re} \sum_{j, k} \sum_{\substack{|\alpha|=m_{j},|\beta|=m_{k}}} a_{\alpha, \beta}^{j, k}(x) \zeta_{\beta}^{k} \overline{\zeta_{\alpha}^{j}} \geq 0
$$

for any numbers $\zeta_{\alpha}^{k}$ at all points $x \in \Omega$.

Theorem 2 can be viewed as a far reaching extension of a well-known theorem of Meyers, who treated the scalar case $m=1$ in [58]. The example given in Sect. 5 of [58] shows that if the coefficients $A_{\alpha \beta}$ are merely bounded, then $p$ has to be close to 2 , even when $\partial \Omega$ is smooth. For higher order operators we make use of an example due to [46] (cf. also the contemporary article by De Giorgi [22]). Specifically, when $m \in \mathbb{N}$ is even, consider the divergence-form equation

$$
\Delta^{\frac{1}{2} m-1} \mathcal{L}_{4} \Delta^{\frac{1}{2} m-1} \mathcal{U}=0 \text { in } \Omega:=\left\{X \in \mathbb{R}^{n}:|X|<1\right\},
$$

where $\mathcal{L}_{4}$ is the fourth order operator

$$
\begin{aligned}
\mathcal{L}_{4}\left(X, D_{X}\right) \mathcal{U}:= & a \Delta^{2} \mathcal{U}+b \sum_{i, j=1}^{n} \Delta\left(\frac{X_{i} X_{j}}{|X|^{2}} \partial_{i} \partial_{j} \mathcal{U}\right)+b \sum_{i, j=1}^{n} \partial_{i} \partial_{j}\left(\frac{X_{i} X_{j}}{|X|^{2}} \Delta \mathcal{U}\right) \\
& +c \sum_{i, j, k, l=1}^{n} \partial_{k} \partial_{l}\left(\frac{X_{i} X_{j} X_{k} X_{l}}{|X|^{4}} \partial_{i} \partial_{j} \mathcal{U}\right) .
\end{aligned}
$$

Obviously, the coefficients of $\mathcal{L}_{4}\left(X, D_{X}\right)$ are bounded, and if the parameters $a, b, c \in$ $\mathbb{R}, a>0$, are chosen such that $b^{2}<a c$ then $\mathcal{L}$ along with $\Delta^{\frac{1}{2} m-1} \mathcal{L}_{4} \Delta^{\frac{1}{2} m-1}$ are strongly elliptic. It has been observed in [46] that the function $\mathcal{U}(X):=|X|^{\theta+m-2} \in$ $W_{2}^{m}(\Omega)$ has $\operatorname{Tr} \mathcal{U} \in C^{\infty}(\partial \Omega)$ and is a weak solution of (1.22) for the choice

$$
\theta:=2-\frac{n}{2}+\sqrt{\frac{n^{2}}{4}-\frac{(n-1)(b n+c)}{a+2 b+c}} .
$$

Thus, if

$$
a:=(n-2)^{2}+\varepsilon, \quad b:=n(n-2), \quad c:=n^{2}, \quad \varepsilon>0,
$$

the strong ellipticity condition is satisfied and $\theta=\theta(\varepsilon)$ becomes

$$
2-n / 2+n \varepsilon^{1 / 2} / 2\left(4(n-1)^{2}+\varepsilon\right)^{1 / 2}
$$

However, $\mathcal{U} \in W_{p}^{m}(\Omega)$ if and only if $p<n /(2-\theta(\varepsilon))$, and the bound $n /(2-\theta(\varepsilon))$ approaches 2 when $\varepsilon \rightarrow 0$. An analogous example can be produced when $m>1$ is 
odd, starting with a sixth order operator $\mathcal{L}_{6}\left(X, D_{X}\right)$ from [46]. In the above context, given that $W_{n}^{1}(\Omega) \hookrightarrow V M O(\Omega)$, it is significant to point out that both for the example in [58], when $n=2$, and for (1.22) when $n \geq 3$, the coefficients have their gradients in weak- $L_{n}$, yet they fail to belong to $W_{n}^{1}(\Omega)$.

We conclude this subsection with a remark pertaining to the presence of lower order terms. More specifically, in view of Theorem 1, a standard perturbation argument (cf., e.g., [35]) proves the following. Assume that

$$
\mathcal{A}\left(X, D_{X}\right) \mathcal{U}:=\sum_{0 \leq|\alpha|,|\beta| \leq m} D^{\alpha}\left(\mathcal{A}_{\alpha \beta}(X) D^{\beta} \mathcal{U}\right), \quad X \in \Omega,
$$

where the principal part of $\mathcal{A}\left(X, D_{X}\right)$ satisfies the hypotheses made in Theorem 1 and the coefficients of the lower order terms are bounded. Then, assuming that (1.20) holds, the Dirichlet problem (1.19) is Fredholm with index zero in the sense that the operator

$$
W_{p}^{m, a}(\Omega) \ni \mathcal{U} \mapsto\left(\mathcal{A}\left(X, D_{X}\right) \mathcal{U},\left\{\partial^{k} \mathcal{U} / \partial v^{k}\right\}_{0 \leq k \leq m-1}\right) \in W_{p}^{-m, a}(\Omega) \oplus \dot{W}_{p}^{m-1+s}(\partial \Omega)
$$

is Fredholm. Furthermore, the estimate

$$
\|\mathcal{U}\|_{W_{p}^{m, a}(\Omega)} \leq C\left(\|\mathcal{F}\|_{W_{p}^{-m, a}(\Omega)}+\|g\|_{\dot{W}_{p}^{m-1+s}(\partial \Omega)}+\|\mathcal{U}\|_{L_{p}(\Omega)}\right)
$$

holds for any solution $\mathcal{U} \in W_{p}^{m, a}(\Omega)$ of (1.19).

\subsection{Comments on tools used in the proof of Theorem 1}

One difficulty linked with the case $m>1$ arises from the way the norm

$$
\left(\sum_{|\alpha| \leq m} \int_{\Omega}\left|D^{\alpha} \mathcal{U}(X)\right|^{p} \rho(X)^{p a} d X\right)^{1 / p}
$$

behaves under a change of variables

$$
\varkappa: \Omega=\left\{\left(X^{\prime}, X_{n}\right): X_{n}>\varphi\left(X^{\prime}\right)\right\} \rightarrow \mathbb{R}_{+}^{n}
$$

used to flatten the Lipschitz surface $\partial \Omega$. When $m=1$, a simple bi-Lipschitz change of variables such as $\Omega \ni\left(X^{\prime}, X_{n}\right) \mapsto\left(X^{\prime}, X_{n}-\varphi\left(X^{\prime}\right)\right) \in \mathbb{R}_{+}^{n}$ will do, but matters are considerably more subtle in the case $m>1$. The extension operator used in [51] was introduced by Nečas (in a different context; cf. p. 188 in [61]) and rediscovered by Maz'ya and Shaposhnikova [56] (see also [57], and later by Dahlberg et al. (cf. [16] and the discussion in [19]), and Hofmann and Lewis [33].

The extension operator in question is defined in the following way. Fix a smooth, radial, decreasing, even, non-negative function $\zeta$ in $\mathbb{R}^{n-1}$ such that $\zeta(t)=0$ for $|t| \geq 1$ 
and

$$
\int_{\mathbb{R}^{n-1}} \zeta(t) d t=1
$$

(For example, $\zeta(t):=c \exp \left(-1 /\left(1-|t|^{2}\right)_{+}\right)$for a suitable $c$.) Define the extension operator $T$ by

$$
(T \varphi)\left(x^{\prime}, x_{n}\right):=\int_{\mathbb{R}^{n-1}} \zeta(t) \varphi\left(x^{\prime}+x_{n} t\right) d t, \quad\left(x^{\prime}, x_{n}\right) \in \mathbb{R}_{+}^{n},
$$

acting on functions $\varphi$ from $L_{1, l o c}\left(\mathbb{R}^{n-1}\right)$.

The following two estimates provide useful properties of the operator $T$.

(i) For each multi-indices $\alpha$ with $|\alpha|>1$ there exists $c>0$ such that

$$
\left|D_{x}^{\alpha}(T \varphi)(x)\right| \leq c x_{n}^{1-|\alpha|}\|\nabla \varphi\|_{B M O\left(\mathbb{R}^{n-1}\right)}, \quad \forall x=\left(x^{\prime}, x_{n}\right) \in \mathbb{R}_{+}^{n} .
$$

(ii) If $\nabla_{x^{\prime}} \varphi \in B M O\left(\mathbb{R}^{n-1}\right)$ then $\nabla(T \varphi) \in B M O\left(\mathbb{R}_{+}^{n}\right)$ and

$$
\|\nabla(T \varphi)\|_{B M O\left(\mathbb{R}_{+}^{n}\right)} \leq c\left\|\nabla_{x^{\prime}} \varphi\right\|_{B M O\left(\mathbb{R}^{n-1}\right)} .
$$

Another ingredient in the proof of Theorem 1 is the derivation of estimates for $D_{x}^{\alpha} D_{y}^{\beta} G(x, y)$ where $G$ is the Green matrix of the operator

$$
L\left(D_{x}\right)=\sum_{|\alpha|=2 m} A_{\alpha} D_{x}^{\alpha}
$$

i.e. the unique solution of the boundary-value problem

$$
\begin{cases}L\left(D_{x}\right) G(x, y)=\delta(x-y) I_{l} & \text { for } x \in \mathbb{R}_{+}^{n}, \\ \left(\frac{\partial^{j}}{\partial x_{n}^{j}} G\right)\left(\left(x^{\prime}, 0\right), y\right)=0 I_{l} & \text { for } x^{\prime} \in \mathbb{R}^{n-1}, 0 \leq j \leq m-1\end{cases}
$$

where $y \in \mathbb{R}_{+}^{n}$ is regarded as a parameter. The methods employed in earlier works are based on explicit representation formulas for $G(x, y)$ and cannot be adapted to the case of non-symmetric, complex coefficient, higher order systems. The approach in [51] consists of proving that the residual part $R(x, y):=G(x, y)-\Phi(x-y)$, where $\Phi$ is a fundamental matrix for $L\left(D_{x}\right)$, has the property

$$
\left\|D_{x}^{\alpha} D_{y}^{\beta} R(x, y)\right\| \leq C|x-\bar{y}|^{-n}
$$

for $|\alpha|=|\beta|=m, x, y \in \mathbb{R}_{+}^{n}$, where $\bar{y}:=\left(y^{\prime},-y_{n}\right)$ is the reflection of the point $y \in \mathbb{R}_{+}^{n}$ with respect to $\partial \mathbb{R}_{+}^{n}$. 


\section{The Stokes system}

By $B_{p, q}^{s}\left(\mathbb{R}^{n}\right)$ we denote the space of functions in $\mathbb{R}^{n}$ having the finite norm

$$
\|u\|_{B_{p, q}^{s}\left(\mathbb{R}^{n}\right)}=\left(\int_{\mathbb{R}^{n}}\left\|\Delta_{h} \nabla_{[s]} u\right\|_{L_{p}\left(\mathbb{R}^{n}\right)}^{q}|h|^{-n-q\{s\}} d h\right)^{1 / q}+\|u\|_{W_{p}^{[s]}\left(\mathbb{R}^{n}\right)},
$$

where $\{s\}>0, p, q \geq 1, \Delta_{h} v=v(\cdot+h)-v(\cdot)$, and $\nabla_{[s]}$ is the vector of all derivatives of order $[s]$. For $p=q$ we use the notation $B_{p}^{s}\left(\mathbb{R}^{n}\right)$.

The Besov scale $B_{p, q}^{s}(\Omega)$ is defined by restricting the (tempered) distributions from the corresponding spaces in $\mathbb{R}^{n}$ to the open set $\Omega$. Also, $B_{p, q}^{s}(\partial \Omega)$ stands for the Besov class on the Lipschitz manifold $\partial \Omega$, obtained by transporting (via a partition of unity and pull-back) the standard scale $B_{p, q}^{s}\left(\mathbb{R}^{n-1}\right)$.

\subsection{Weak solvability in Besov and Triebel-Lizorkin spaces}

Here we discuss an extension of the material described in Sect. 1 to the Dirichlet problem for the Stokes system, following the article [52]. This problem is considered in more general function scales than in Sect. 1.

Consider the Stokes system in an arbitrary bounded Lipschitz domain $\Omega \subset \mathbb{R}^{n}$, $n \geq 2$,

$$
\begin{aligned}
& \Delta u-\nabla \pi=f \in B_{p, q}^{s+\frac{1}{p}-2}(\Omega), \quad \operatorname{div} u=g \in B_{p, q}^{s+\frac{1}{p}-1}(\Omega), \\
& u \in B_{p, q}^{s+\frac{1}{p}}(\Omega), \quad \pi \in B_{p, q}^{s+\frac{1}{p}-1}(\Omega), \quad \operatorname{Tr} u=h \in B_{p, q}^{s}(\partial \Omega),
\end{aligned}
$$

subject to the (necessary) compatibility condition

$$
\int_{\partial \mathcal{O}}\langle v, h\rangle d \sigma=\int_{\mathcal{O}} g(X) d X, \quad \text { for every component } \mathcal{O} \text { of } \Omega .
$$

When $\partial \Omega$ is sufficiently smooth (at least of the class $C^{2}$ ), the problem (2.2) was studied in many papers, first in Sobolev spaces with an integer amount of smoothness by Solonnikov [67], Cattabriga [13], Temam [69], Giga [31], Dautray and Lions [20], among others. This has been subsequently extended by Amrouche and Girault [5] to the case when $\partial \Omega \in C^{1,1}$ and, further, by Galdi et al. [30] when $\partial \Omega$ is Lipschitz, with a small Lipschitz constant. The case when $\Omega$ is a polygonal domain in $\mathbb{R}^{2}$, or a polyhedral domain in $\mathbb{R}^{3}$ also has a rich history. An extended account of this field of research can be found in the monograph by Kozlov et al. [45] which also contains references to earlier work. Among recent publications we mention the paper by Maz'ya and Rossmann [53] as well as their book [54]. Lipschitz and $C^{1}$ subdomains of Riemannian manifolds were treated in the paper by Dindoš and Mitrea [26], and the paper by Mitrea and Taylor [60]. 
The principal result in [52] on solutions of the problem (2.2) holds under mild conditions on the normal $v$ to the boundary of the Lipschitz graph domain, similar to those in Theorem 1 in Sect. 1.3. It runs as follows.

Theorem 3 Assume that

$$
\frac{n-1}{n}<p \leq \infty, \quad 0<q \leq \infty, \quad(n-1)\left(\frac{1}{p}-1\right)_{+}<s<1 .
$$

If there exists $\delta>0$ which depends only on the Lipschitz constant of $\Omega$ and the exponent $p$, with the property that if $\{v\}_{O s c(\partial \Omega)}<\delta$, then the problem (2.2) is well-posed (with uniqueness modulo locally constant functions in $\Omega$ for the pressure). There exists a finite, positive constant $C=C(\Omega, p, q, s, n)$ such that

$$
\begin{aligned}
& \|u\|_{B_{p, q}^{s+\frac{1}{p}}(\Omega)}+\inf _{c}\|\pi-c\|_{B_{p, q}^{s+\frac{1}{p}-1}(\Omega)} \\
& \leq C\left(\|f\|_{B_{p, q}^{s+\frac{1}{p}-2}(\Omega)}+\|g\|_{B_{p, q}^{s+\frac{1}{p}-1}(\Omega)}+\|h\|_{B_{p, q}^{s}(\partial \Omega)}\right),
\end{aligned}
$$

where the infimum is taken over all locally constant functions $c$ in $\Omega$.

Moreover, an analogous well-posedness result holds for the Triebel-Lizorkin scale (for its definition see, for example, the book by Runst and Sickel [64]), i.e. for the problem

$$
\begin{aligned}
& \Delta u-\nabla \pi=f \in F_{p, q}^{s+\frac{1}{p}-2}(\Omega), \quad \operatorname{div} u=g \in F_{p, q}^{s+\frac{1}{p}-1}(\Omega), \\
& u \in F_{p, q}^{s+\frac{1}{p}}(\Omega), \quad \pi \in F_{p, q}^{s+\frac{1}{p}-1}(\Omega), \quad \operatorname{Tr} u=h \in B_{p, q}^{s}(\partial \Omega) .
\end{aligned}
$$

This time, in addition to the previous conditions imposed on the indices $p, q$, it is also assumed that $p, q<\infty$.

It should be noted that conditions (2.4) describe the largest range of indices $p, q, s$ for which the Besov spaces $B_{p, q}^{s}(\partial \Omega)$ can be meaningfully defined on the Lipschitz manifold $\partial \Omega$.

\subsection{Dirichlet data in $L_{p}(\Omega)$}

We say a few words about the Dirichlet problem for the multi-dimensional Stokes system with $L_{p}$ boundary data which is not touched upon in [52]. Following the paper by Dindoš and Maz'ya [25], we shall speak about both the Lamé system (with the Poisson ratio $\alpha<1 / 2$ ) and the Stokes system (with $\alpha=1 / 2$ ).

Let us consider a bounded domain $\Omega$ in $\mathbb{R}^{n}, n \geq 3$, and the system

$$
\Delta u-\nabla \pi=0, \quad \operatorname{div} u+(1-2 \alpha) \pi=0 \quad \text { in } \Omega,
$$


complemented by the condition

$$
\operatorname{Tr} u=h .
$$

The boundary values $\operatorname{Tr} u$ are understood in nontangential sense, that is in the sense of the limit

$$
\operatorname{Tr} u(x)=\lim _{y \rightarrow x, y \in \Gamma(x)} u(y)
$$

over a collection of interior nontangential cones $\Gamma(x)$ with vertices at $x \in \partial \Omega$. Let $u^{*}$ be the classical nontangential maximal function defined as

$$
u^{*}(x)=\sup _{y \in \Gamma(x)}|u(y)| \quad \text { for all } x \in \partial \Omega .
$$

The class of solutions to (2.6), (2.7) is described by the inclusion

$$
u^{*} \in L_{p}(\partial \Omega) .
$$

One says that problem (2.6), (2.7) is $L_{p}$ solvable if for all vector fields $h \in L_{p}(\partial \Omega)$ there is a pair $(u, \pi)$ satisfying (2.6)-(2.8) and moreover, for some $C>0$ independent of $h$, the estimate

$$
\left\|u^{*}\right\|_{L_{p}(\partial \Omega)} \leq C\|h\|_{L_{p}(\partial \Omega)}
$$

holds. Furthermore, the problem (2.6), (2.7) is said to be solvable for continuous data if, for all $h \in C(\partial \Omega)$ the vector field $u$ belongs to $C(\bar{\Omega})$ and the estimate

$$
\|u\|_{C(\bar{\Omega})} \leq C\|h\|_{C(\partial \Omega)}
$$

holds.

The $L_{p}$ solvability of the Dirichlet problem for the Stokes system is established by Shen [66] for all $p \in(2-\varepsilon(\Omega), \infty$ ] provided $\Omega$ is a three-dimensional Lipschitz graph domain. In [25], the problem (2.6), (2.7) is considered on domains in $\mathbb{R}^{n}, n \geq 3$, with isolated conical singularity (not necessary a Lipschitz graph) and the authors prove its solvability for all $p \in(2-\varepsilon(\Omega), \infty]$ as well as its solvability in $C(\bar{\Omega})$ for the data in $C(\partial \Omega)$. This seems to be a strong indication that the range $p \in(2-\varepsilon(\Omega), \infty]$ should hold also for Lipschitz graph domains. However, there is no such result for $n>3$.

\subsection{Lipschitz continuous solutions}

We cite a regularity result for solutions of the Dirichlet problem for the Stokes system in a plane convex domain obtained by Kozlov and Maz'ya [44]:

$$
\begin{aligned}
& -\Delta u+\nabla \pi=f, \quad \operatorname{div} u=0 \quad \text { in } \Omega, \\
& \operatorname{Tr} u=0 \text { on } \partial \Omega,
\end{aligned}
$$

where $f \in W_{2}^{-1}(\Omega)$ and $(u, \pi) \in \stackrel{\circ}{W}_{2}^{1}(\Omega) \times L_{2}(\Omega)$. 
Theorem 4 Let $\Omega$ be a bounded convex two-dimensional domain and let $f \in L_{q}(\Omega)$ for some $q>2$. Then the velocity vector $u \in \stackrel{\circ}{W}_{2}^{1}(\Omega)$ admits the estimate

$$
\|\nabla u\|_{L_{\infty}(\Omega)} \leq C\|f\|_{L_{q}(\Omega)},
$$

where $C$ depends only on $\Omega$.

A direct consequence of this result for the nonlinear Navier-Stokes system is as follows.

Corollary 1 Let $(u, \pi) \in \stackrel{\circ}{W}_{2}^{1}(\Omega) \times L_{2}(\Omega)$ solve the Dirichlet problem

$$
\begin{aligned}
-\Delta u+\nabla \pi+\sum_{k=1}^{2} u_{k} \partial_{k} u & =f, \quad \text { div } u=0 \text { in } \Omega, \\
u & =0 \quad \text { on } \partial \Omega,
\end{aligned}
$$

where $f \in W_{2}^{-1}(\Omega)$. Let $\Omega$ be a bounded convex two-dimensional domain and let $f \in L_{q}(\Omega)$ for some $q>2$. Then the velocity vector $u \in \stackrel{\circ}{W}_{2}^{1}(\Omega)$ belongs to the class $C^{0,1}(\bar{\Omega})$ of functions uniformly satisfying the Lipschitz condition on $\bar{\Omega}$.

A result of the same nature was obtained by Maz'ya [48] for solutions of the Neumann problem for the Poisson equation in arbitrary convex $n$-dimensional domains. Let $\Omega$ be a bounded convex domain in $\mathbb{R}^{n}$ and let $W_{p}^{l}(\Omega)$ stand for the Sobolev space of functions in $L_{p}(\Omega)$ with distributional derivatives of order $l$ in $W_{p}^{l}(\Omega)$. By $L_{p, \perp}(\Omega)$ and $W_{p, \perp}^{l}(\Omega)$ we denote the subspaces of functions in $L_{p}(\Omega)$ and $W_{p}^{l}(\Omega)$ subject to

$$
\int_{\Omega} v d x=0 .
$$

Theorem 5 Let $f \in L_{q, \perp}(\Omega)$ with a certain $q>n$ and let $u$ be the unique weak solution in $W_{2, \perp}^{1}(\Omega)$ of the Neumann problem

$$
\begin{aligned}
-\Delta u & =f \text { in } \Omega, \\
\frac{\partial u}{\partial v} & =0 \quad \text { on } \partial \Omega,
\end{aligned}
$$

where $v$ is the unit outward normal to $\partial \Omega$. Then the estimate

$$
\|\nabla u\|_{L_{\infty}(\Omega)} \leq C\|f\|_{L_{q}(\Omega)}
$$

holds with $C$ independent of $f$. 
As a particular case of a result obtained by Cianchi and Maz'ya [14] for a class of nonlinear equations, one can replace the $L_{q}$-norm on the right-hand side by the Lorentz norm $\|f\|_{L^{n, 1}(\Omega)}$ which is the best possible majorant among those formulated in terms of Lorentz spaces. Similar facts for Lamé and Stokes systems with boundary conditions different from those of Dirichlet are unknown.

\section{Higher regularity of solutions}

\subsection{Preliminaries}

In this section we survey some applications of Sobolev multipliers to the question of higher regularity in fractional Sobolev spaces of solutions to boundary value problems for higher order elliptic equations in a Lipschitz domain. Since there is no higher regularity of solutions for general Lipschitz graph domains, one is forced to find a proper subclass of Lipschitz domains for which the regularity holds.For domains of this subclass we developed a solvability and regularity theory analogous to the classical one for smooth domains. We also showed that the chosen subclass of Lipschitz domains is best possible in a certain sense. This was done in [55] (see also Chap. 14 in [57]). We give a short description of that theory.

Let $\Omega$ be a domain in $\mathbb{R}^{n}$ with compact closure. Throughout this section we assume that for any point of the boundary $\partial \Omega$ there exists a Cartesian coordinate system $(x, y)$, $x \in \mathbb{R}^{n-1}, y \in \mathbb{R}^{1}$, a neighbourhood $U$ and a Lipschitz function $\varphi$ such that

$$
U \cap \Omega=U \cap\left\{z=(x, y): x \in \mathbb{R}^{n-1}, y>\varphi(x)\right\} .
$$

Let $B_{p}^{l-1 / p}\left(\mathbb{R}^{n-1}\right), l=1,2, \ldots$, denote the completion of the space $C_{0}^{\infty}\left(\mathbb{R}^{n-1}\right)$ in the norm

$$
\left(\int_{\mathbb{R}^{n-1}} \int_{\mathbb{R}^{n-1}}\left|\nabla_{l-1} u(t)-\nabla_{l-1} u(x)\right|^{p}|x-t|^{2-n-p} d x d t\right)^{1 / p}+\|u\|_{L_{p}\left(\mathbb{R}^{n-1}\right)} .
$$

Replacing $\mathbb{R}^{n-1}$ by $\partial \Omega$ one arrives at the definition of the space $B_{p}^{l-1 / p}(\partial \Omega)$.

We need the space of Sobolev multipliers $M\left(W_{p}^{h}(\Omega) \rightarrow W_{p}^{l}(\Omega)\right)$, that is the class of functions $\gamma$ such that $\gamma u \in W_{p}^{l}(\Omega)$ for all $u \in W_{p}^{h}(\Omega)$. The space $M\left(W_{p}^{h}(\Omega) \rightarrow\right.$ $\left.W_{p}^{l}(\Omega)\right)$ is endowed with the norm

$$
\|\gamma\|_{M\left(W_{p}^{h}(\Omega) \rightarrow W_{p}^{l}(\Omega)\right)}=\sup \left\{\|\gamma u\|_{W_{p}^{l}(\Omega)}:\|u\|_{W_{p}^{h}(\Omega)} \leq 1\right\} .
$$

The notation $M S$ is used instead of $M(S \rightarrow S)$, where $S$ is a Banach function space. Properties and applications of Sobolev multipliers are studied in detail in [57].

We introduce the essential norm of a function $\gamma \in M\left(W_{p}^{h}(\Omega) \rightarrow W_{p}^{l}(\Omega)\right)$ :

$$
\operatorname{ess}\|\gamma\|_{M\left(W_{p}^{h}(\Omega) \rightarrow W_{p}^{l}(\Omega)\right)}=\inf _{\{T\}}\|\gamma-T\|_{W_{p}^{h}(\Omega) \rightarrow W_{p}^{l}(\Omega)},
$$


where $\{T\}$ is the set of all compact operators: $W_{p}^{h}(\Omega) \rightarrow W_{p}^{l}(\Omega)$.

Analytic two-sided and one-sided estimates for the norm (3.2) and the essential norm (3.3) can be found in [57].

\subsection{Subclasses of Lipschitz graph domains}

Let $\Omega$ be a bounded Lipschitz graph domain. We introduce the class $M_{p}^{l-1 / p}(l=$ $2,3, \ldots$ ) of boundaries $\partial \Omega$, satisfying the following condition. For every point of $\partial \Omega$ there exists an $n$-dimensional neighborhood in which $\partial \Omega$ is specified (in a certain Cartesian coordinate system) by a function $\varphi$ such that

$$
\nabla \varphi \in M B_{p}^{l-1-1 / p}\left(\mathbb{R}^{n-1}\right)
$$

Furthermore, by definition, $M_{p}^{1-1 / p}$ is the class of bounded Lipschitz graph domains.

We say that $\partial \Omega$ belongs to the class $B_{p}^{l-1 / p}$ if $\partial \Omega$ can be locally specified by a function $\varphi \in B_{p}^{l-1 / p}\left(\mathbb{R}^{n-1}\right)$. Since

$$
M B_{p}^{l-1-1 / p}\left(\mathbb{R}^{n-1}\right) \subset B_{p, \text { loc }}^{l-1-1 / p}\left(\mathbb{R}^{n-1}\right), \quad l \geq 2,
$$

it follows that any bounded domain $\Omega$ with $\partial \Omega \in M_{p}^{l-1 / p}$ satisfies $\partial \Omega \in B_{p}^{l-1 / p}$. (We say that $u \in B_{p, \text { loc }}^{s}\left(\mathbb{R}^{n-1}\right)$ if $u \eta \in B_{p}^{s}\left(\mathbb{R}^{n-1}\right)$ for any $\eta \in C_{0}^{\infty}\left(\mathbb{R}^{n-1}\right)$.)

According to Corollary 4.3.8 in [57], for $p(l-1)>n$ we have

$$
\|\nabla \varphi\|_{M B_{p}^{l-1-1 / p}\left(\mathbb{R}^{n-1}\right)} \sim \sup _{x \in \mathbb{R}^{n-1}}\|\nabla \varphi\|_{B_{p}^{l-1-1 / p}\left(\mathcal{B}_{1}(x)\right)}
$$

where $\mathcal{B}_{r}(x)$ is a ball in $\mathbb{R}^{n-1}$ with radius $r$ and center $x$. Therefore, the classes $M_{p}^{l-1 / p}$ and $B_{p}^{l-1 / p}$ coincide for $p(l-1)>n$.

In our subsequent exposition the following additional condition on $\Omega$ plays an important role.

Let $\delta>0$. We say that $\partial \Omega$ belongs to the class $M_{p}^{l-1 / p}(\delta)$ if for every point $O \in \partial \Omega$ there exists a Cartesian coordinate system $(x, y), x \in \mathbb{R}^{n-1}, y \in \mathbb{R}^{1}$, a neighborhood $U$ and a special Lipschitz domain $G=\left\{z=(x, y): x \in \mathbb{R}^{n-1}, y>\varphi(x)\right\}$ such that $U \cap \Omega=U \cap G$ and

$$
\|\nabla \varphi\|_{M B_{p}^{l-1-1 / p}\left(\mathbb{R}^{n-1}\right)} \leq \delta
$$

Obviously, the boundaries in $M_{p}^{l-1 / p}(\delta)$ belong to the class $M_{p}^{l-1 / p}$ and, therefore, to the class $B_{p}^{l-1 / p}$.

The following assertion gives a local characterization of the class $M_{p}^{l-1 / p}(\delta)$ (see Sect. 14.7 in [57]). In its statement we use the notion of the $(p, j)$-capacity of a 
compact set $e$ in $\mathbb{R}^{n-1}$ :

$$
C_{p, j}(e)=\inf \left\{\|u\|_{W_{p}^{j}\left(\mathbb{R}^{n-1}\right)}^{p}: u \in C_{0}^{\infty}\left(\mathbb{R}^{n-1}\right), u \geq 1 \text { on } e\right\} .
$$

For various properties of this capacity see the books by Maz'ya [47] and Adams and Hedberg [1].

Proposition 3 Let $p(l-1) \leq n$. The class $M_{p}^{l-1 / p}(\delta)$ admits the following equivalent description. For any point $O \in \partial \Omega$ there exists a Cartesian coordinate system $(x, y), x \in \mathbb{R}^{n-1}, y \in \mathbb{R}^{1}$, a neighborhood $U$ and a special Lipschitz domain $G=\left\{z=(x, y): x \in \mathbb{R}^{n-1}, y>\varphi(x)\right\}$ such that $U \cap \Omega=U \cap G$ and

$$
\lim _{\varepsilon \rightarrow 0}\left(\sup _{e \subset \mathcal{B}_{\varepsilon}} \frac{\left\|D_{l-1 / p}\left(\varphi ; \mathcal{B}_{\varepsilon}\right)\right\|_{L_{p}(e)}}{\left(C_{p, l-1-1 / p}(e)\right)^{1 / p}}+\|\nabla \varphi\|_{L_{\infty}\left(\mathcal{B}_{\varepsilon}\right)}\right) \leq c \delta .
$$

Here $\mathcal{B}_{\varepsilon}$ is the ball with centre at $O$ and radius $\varepsilon, c$ is a constant which depends only on $l, p, n$, and

$$
D_{l-1 / p}\left(\varphi ; \mathcal{B}_{\varepsilon}\right)(x)=\left(\int_{\mathcal{B}_{\varepsilon}}\left|\nabla_{l-1} \varphi(x)-\nabla_{l-1} \varphi(y)\right|^{p} \frac{d y}{|x-y|^{n-2+p}}\right)^{1 / p} .
$$

Proposition 3 and properties of the capacity lead to the following sufficient condition formulated in terms of the $(n-1)$-dimensional Lebesgue measure $m_{n-1}$.

Corollary 2 (i) If $n>p(l-1)$ and

$$
\lim _{\varepsilon \rightarrow 0}\left(\sup _{e \subset \mathcal{B}_{\varepsilon}} \frac{\left\|D_{l-1 / p}\left(\varphi ; \mathcal{B}_{\varepsilon}\right)\right\|_{L_{p}(e)}}{\left(m_{n-1} e\right)^{[n-p(l-1)] /(n-1) p}}+\|\nabla \varphi\|_{L_{\infty}\left(\mathcal{B}_{\varepsilon}\right)}\right)<c \delta,
$$

then $\partial \Omega \in M_{p}^{l-1 / p}(\delta)$.

(ii) If $n=p(l-1)$ and

$\lim _{\varepsilon \rightarrow 0}\left(\sup _{e \subset \mathcal{B}_{\varepsilon}}\left\|D_{l-1 / p}\left(\varphi ; \mathcal{B}_{\varepsilon}\right)\right\|_{L_{p}(e)}\left|\log \left(m_{n-1} e\right)\right|^{(p-1) / p}+\|\nabla \varphi\|_{L_{\infty}\left(\mathcal{B}_{\varepsilon}\right)}\right)<c \delta$,

then $\partial \Omega \in M_{p}^{l-1 / p}(\delta)$.

Now we present another test for the inclusion of a function into $M_{p}^{l-1 / p}(\delta)$ involving the Besov space $B_{q, p}^{m}$.

We say that the boundary of a Lipschitz graph domain $\Omega$ belongs to $B_{q, p}^{l-1 / p}(l=$ $1,2, \ldots$, ) if, for any point of $\partial \Omega$, there exists a neighborhood in which $\partial \Omega$ is specified 
in Cartesian coordinates by a function $\varphi$ satisfying

$$
\int_{\mathbb{R}^{n-1}}\left(\int_{\mathbb{R}^{n-1}}\left|\nabla_{l-1} \varphi(x+h)-\nabla_{l-1} \varphi(x)\right|^{q} d x\right)^{p / q} \frac{d h}{|h|^{n+p-2}}<\infty .
$$

Corollary 3 Let $p(l-1) \leq n$ and let $\Omega$ be a bounded Lipschitz graph domain with $\partial \Omega \in B_{q, p}^{l-1 / p}$, where

$$
q \in[p(n-1) /(p(l-1)-1), \infty] \text { if } p(l-1)<n
$$

and

$$
q \in(p, \infty] \text { if } p(l-1)=n
$$

Further, let $\partial \Omega$ be locally defined in Cartesian coordinates by $y=\varphi(x)$, where $\varphi$ is a function with a Lipschitz constant less than $c \delta$. Then $\partial \Omega \in M_{p}^{l-1 / p}(\delta)$.

Setting $q=\infty$ in Corollary 3, one obtains the simple sufficient condition for the inclusion into $M_{p}^{l-1 / p}(\delta)$ formulated in terms of the modulus of continuity $\omega_{l-1}(t)$ of $\nabla_{l-1} \varphi$ :

$$
\int_{0}^{1}\left(\frac{\omega_{l-1}(t)}{t}\right)^{p} d t<\infty
$$

Since $B_{\infty, p}^{l-1 / p} \subset B_{p}^{l-1 / p}$, it follows that (3.7) is sufficient for $\partial \Omega \in B_{p}^{l-1 / p}$.

\subsection{General elliptic boundary value problems}

Consider either scalar or square matrix differential operators

$$
P\left(z, D_{z}\right)=\sum_{|\alpha| \leq 2 m} a_{\alpha}(z) D_{z}^{\alpha}, \quad P_{j}\left(z, D_{z}\right)=\sum_{|\alpha| \leq k_{j}} a_{\alpha j}(z) D_{z}^{\alpha},
$$

where $z \in \bar{\Omega}, 1 \leq j \leq m$, and $D_{z}=\left(i^{-1} \partial_{z_{1}}, \ldots, i^{-1} \partial_{z_{n}}\right)$. We consider the operator of a boundary value problem $\left\{P ; \operatorname{Tr} P_{1}, \ldots, \operatorname{Tr} P_{m}\right\}$.

The next result is proved essentially in the same manner as Theorem 14.3.1 in [57].

Theorem 6 Suppose that for any neighbourhood $U \subset \mathbb{R}^{n}$ there exist operators

$$
P^{U}\left(D_{z}\right)=\sum_{|\alpha|=2 m} a_{\alpha}^{U} D_{z}, \quad P_{j}^{U}\left(D_{z}\right)=\sum_{|\alpha|=k_{j}} a_{\alpha j}^{U} D_{z}
$$


with constant coefficients such that $\left\{P^{U} ; \operatorname{Tr} P_{j}^{U}\right\}$ is the operator of an elliptic boundary value problem in the half-space $\left\{z=(x, y): x \in \mathbb{R}^{n-1}, y \geq 0\right\}$.

Let

$$
\begin{aligned}
& \sum_{|\alpha|=2 m}\left\|a_{\alpha}(z)-a_{\alpha}^{U}\right\|_{L_{\infty}(U \cap \Omega)} \leq \delta, \\
& \sum_{|\alpha| \leq 2 m} \operatorname{ess}\left\|a_{\alpha}\right\|_{M\left(W_{p}^{l-|\alpha|}(\Omega) \rightarrow W_{p}^{l-2 m}(\Omega)\right)} \leq \delta,
\end{aligned}
$$

wherel is integer, $l \geq 2 m, 1<p<\infty$. The constant $\delta$ here and elsewhere is supposed to be small in comparison with the norms of the inverse operators $\left\{P^{U} ; \operatorname{Tr} P_{j}^{U}\right\}^{-1}$ for all $U$. Further, let the coefficients $a_{\alpha j}$ satisfy conditions similar to (3.9), with $2 m$ replaced by $k_{j}$.

Let the boundary $\partial \Omega$ belong to the class $M_{p}^{l-1 / p}(\delta)$ if $p(l-1) \leq n$ or to the class $B_{p}^{l-1 / p}$ if $p(l-1)>n$, in either case with $1<p<\infty$.

Then the operator

$$
\left\{P ; \operatorname{Tr} P_{j}\right\}: W_{p}^{l}(\Omega) \rightarrow W_{p}^{l-2 m}(\Omega) \times \prod_{j=1}^{m} B_{p}^{l-k_{j}-1 / p}(\partial \Omega)
$$

is Fredholm. In particular, for all $u \in W_{p}^{l}(\Omega)$ the a priori estimate

$$
\|u\|_{W_{p}^{l}(\Omega)} \leq c\left(\|P u\|_{W_{p}^{l-2 m}(\Omega)}+\sum_{j=1}^{m}\left\|\operatorname{Tr} P_{j} u\right\|_{B_{p}^{l-k_{j}-1 / p}(\partial \Omega)}+\|u\|_{L_{1}(\Omega)}\right)
$$

holds; the last norm in the right-hand side can be omitted in the case of a unique solution.

Note that even the most restrictive sufficient condition (3.7) on the domain for Theorem 6 to hold is sharp. Let $\omega$ be an increasing function in $C[0,1]$ such that $\omega(0)=0$,

$$
\delta \int_{\delta}^{1} \omega(t) \frac{d t}{t^{2}}+\int_{0}^{\delta} \omega(t) \frac{d t}{t} \leq c \omega(\delta)
$$

and

$$
\int_{0}^{1}\left(\frac{\omega(t)}{t}\right)^{p} d t=\infty
$$

It was shown in Sect. 4.4.3 of [57] that one can construct a function $\varphi$ on $\mathbb{R}^{n-1}$ such that 
(i) the modulus of continuity of $\nabla_{l-1} \varphi$ does not exceed $c \omega$ with $c=$ const;

(ii) supp $\varphi \subset Q_{2 \pi}$, where $Q_{d}=\left\{x \in \mathbb{R}^{n-1}:\left|x_{i}\right|<d\right\}$;

(iii) $\varphi \notin B_{p}^{l-1 / p}\left(\mathbb{R}^{n-1}\right)$.

Given $\varphi$, one can construct a bounded domain $\Omega$ in $\mathbb{R}^{n}$ such that the Neumann problem

$$
\Delta v-v=g \text { in } \Omega, \quad \partial v / \partial v=h \quad \text { on } \partial \Omega
$$

with $g \in W_{p}^{l-2}(\Omega)$ and $h \in B_{p}^{l-1-1 / p}(\partial \Omega)$ may fail to be solvable in $W_{p}^{l}(\Omega)$.

Next we describe conditions on the coefficients of (3.8) which are equivalent to those formulated in Theorem 6 and follow from the results in [57], Ch. 7. In its formulation, we use the notion of capacity of a compact set in $\mathbb{R}^{n}$ defined similarly to (3.5).

Corollary 4 Conditions (3.9) in Theorem 6 can be stated as follows:

(i) The coefficients $a_{\alpha}$ with $|\alpha|=2 m$ are in the class $W_{p}^{l-2 m}(\Omega)$ if $p(l-2 m)>n$ and satisfy the inequality

$$
\left\|a_{\alpha}(z)-a_{\alpha}^{U}\right\|_{L_{\infty}(U \cap \Omega)}+\lim _{\varepsilon \rightarrow 0} \sup _{\{e \subset \Omega: \operatorname{diam}(e) \leq \varepsilon\}} \frac{\left\|\nabla_{l-2 m} a_{\alpha}\right\|_{L_{p}(e)}}{\left(C_{p, l-2 m}(e)\right)^{1 / p}} \leq \delta
$$

if $p(l-2 m) \leq n$;

(ii) The coefficients $a_{\alpha}$ with $|\alpha|<2 m$ are in the class $W_{p}^{l-2 m}(\Omega)$ if $p(l-|\alpha|)>n$ and satisfy the inequality

$$
\lim _{\varepsilon \rightarrow 0}\left(\sup _{\{e \subset \Omega: \operatorname{diam}(e) \leq \varepsilon\}} \frac{\left\|\nabla_{l-2 m} a_{\alpha}\right\|_{L_{p}(e)}}{\left(C_{p, l-|\alpha|}(e)\right)^{1 / p}}+\sup _{x \in \Omega, \rho \leq \varepsilon} \rho^{2 m-|\alpha|-n / p}\left\|a_{\alpha}\right\|_{L_{p}\left(B_{\rho}(x) \cap \Omega\right)}\right) \leq \delta
$$

if $p(l-|\alpha|) \leq n$. Here $B_{\rho}(x)$ is a ball in $\mathbb{R}^{n}$ with radius $\rho$ and center $x$.

Various other sufficient conditions for (3.9) follow from the results in [57].

\subsection{The Dirichlet problem in terms of traces}

Let us first consider the Dirichlet problem as a particular case of the general boundary value problem dealt with in Sect. 3.3. We write the scalar or square matrix elliptic operator $P$ in the form

$$
P u=\sum_{|\alpha|,|\beta| \leq m}(-1)^{|\alpha|} \partial^{\alpha}\left(A_{\alpha \beta}(z) \partial^{\beta} u\right)
$$

where $\partial=\left(\partial_{x_{1}}, \ldots, \partial_{x_{n}}\right)$. Suppose that the coefficients $A_{\alpha \beta}$ are in $C^{l-m}(\bar{\Omega}), l \geq m$, and that the coercivity condition

$$
\operatorname{Re} \int_{\Omega} \sum_{|\alpha|=|\beta|=m} A_{\alpha \beta}(z) \partial^{\alpha} u \overline{\partial^{\beta} u} d z \geq c\|u\|_{W_{2}^{m}(\Omega)}^{2}
$$


holds for $u \in C_{0}^{\infty}(\Omega)$. Assume that $\Omega$ is a Lipschitz graph domain.

We introduce a sufficiently small finite open covering $\{U\}$ of $\bar{\Omega}$ and a corresponding partition of unity $\left\{\zeta_{U}\right\}$. Let

$$
P_{j U}=\partial^{j-1} / \partial y^{j-1}, j=1, \ldots, m \quad \text { if } U \cap \partial \Omega \neq \varnothing
$$

and

$$
P_{j U}=0 \quad \text { if } U \cap \partial \Omega=\varnothing .
$$

The Dirichlet boundary conditions will be prescribed by the operators

$$
P_{j}=\sum_{U} \zeta_{U} P_{j U}
$$

We give a formulation of the Dirichlet problem. Let us look for a function $u \in$ $W_{p}^{l}(\Omega)$ such that

$$
P u=f \quad \text { in } \Omega, \quad \operatorname{Tr} P_{j} u=f_{j} \quad \text { on } \partial \Omega, \quad j=1, \ldots, m,
$$

where $f$ and $f_{j}$ are functions in $W_{p}^{l-2 m}(\Omega)$ and $B_{p}^{l+1-j-1 / p}(\partial \Omega)$ respectively.

Here is the principal result relating the problem (3.15) borrowed from Subsec. 14.5.4 in [57].

Theorem 7 Let any of the following conditions hold:

( $\alpha) m=1, p(l-1) \leq n ; \partial \Omega \in M_{p}^{l-1 / p}(\delta)$, where $\delta$ depends on the coefficients of the principal homoheneous parts of $P$ and $P_{j}$;

( $\beta) ~ m=1, p(l-1)>n ; \partial \Omega \in B_{p}^{l-1 / p}$;

( $\gamma) m>1, \partial \Omega \in M_{p}^{l-1 / p}$ and $\partial \Omega$ is locally defined by equations of the form $y=\varphi(x)$, where $\nabla \varphi \in M B_{p}^{l-1-1 / p}\left(\mathbb{R}^{n-1}\right)$ and $\varphi$ is a function with a small Lipschitz constant $\left(\right.$ for $p(l-1)>n$, this is equivalent to $\left.\partial \Omega \in B_{p}^{l-1 / p}\right)$.

Then the operator

$$
\left\{P ; \operatorname{Tr} P_{j}\right\}: W_{p}^{l}(\Omega) \rightarrow W_{p}^{l-2 m}(\Omega) \times \prod_{j=1}^{m} B_{p}^{l+1-j-1 / p}(\partial \Omega)
$$

is an isomorphism.

An example in Subsection 14.6.1 of [57] shows that for $p(l-1) \leq n$ and for $m=1$ the condition $\partial \Omega \in M_{p}^{l-1 / p}(\delta)$ in part $(\alpha)$ of Theorem 7 cannot be replaced by the assumption that $\partial \Omega$ belongs to the class $M_{p}^{l-1 / p} \cap C^{l-1}$. To be precise, a domain $\Omega$ is constructed with $\partial \Omega \in M_{2}^{3 / 2} \cap C^{1}$ for which the problem

$$
-\Delta u=f \quad \text { in } \Omega, \quad \operatorname{Tr} u=0 \text { on } \partial \Omega
$$


is not generally solvable in $W_{2}^{2}(\Omega)$ for $f \in L_{2}(\Omega)$. This means that the smallness of the seminorm $\|\nabla \varphi\|_{M B_{2}^{1 / 2}\left(\mathbb{R}^{n-1}\right)}$ in the definition of $M_{2}^{3 / 2}(\delta)$ is essential for the solvability of problem $(3.16)$ in $W_{2}^{2}(\Omega)$.

The next assertion, which follows directly from the Implicit Function Theorem 9.5.2 in [57], shows that the condition $\partial \Omega \in B_{p}^{l-1 / p}$ with $p(l-1)>n$ is necessary for the solvability of problem (3.15) in $W_{p}^{l}(\Omega)$ for the operator $P$ of higher than second order.

Theorem 8 Let $\Omega$ be a bounded Lipschitz domain and let $l$ be integer, $l \geq 2 m$, $p(l-1)>n, 1<p<\infty$, and $m>1$. If there exists a solution $u \in W_{p}^{l}(\Omega)$ of the problem

$$
P u=0 \text { in } \Omega, \operatorname{Tr} u=0, \quad \operatorname{Tr} P_{2} u=1, \operatorname{Tr} P_{j} u=0, j=3, \ldots, m,
$$

then $\partial \Omega \in B_{p}^{l-1 / p}$.

Under the additional assumption $\partial \Omega \in C^{l-2,1}$, the necessity of the inclusion $\partial \Omega \in$ $B_{p}^{l-1 / p}$ for $p(l-1) \leq n$ is proved in Subsection 14.6.2 of [57].

Theorem 9 Let $\partial \Omega$ be in the class $C^{l-2,1}$ and let $l$ be integer, $l \geq 2 m, p(l-1) \leq n$, $1<p<\infty$, and $m>1$. If there exists a solution $u \in W_{p}^{l}(\Omega)$ of problem (3.17), then $\partial \Omega \in B_{p}^{l-1 / p}$.

A similar result for the second order operator $P$ in Subsection 14.6.2 of [57] runs as follows.

Theorem 10 Let $l$ be integer, $l \geq 2,1<p<\infty, m=1$, and $P 1 \leq 0$. Let $\Omega$ be a domain with $\partial \Omega \in C^{1}$ and let the modulus of continuity $\omega_{1}$ of the normal to $\partial \Omega$ satisfy the Dini condition

$$
\int_{0}^{1} \omega_{1}(t) \frac{d t}{t}
$$

If, for a nonpositive function $f \in C_{0}^{\infty}(\Omega)$, there exists a solution $u \in W_{p}^{l}(\Omega)$ of the problem

$$
P u=f \quad \text { in } \Omega, \quad \operatorname{Tr} u=0
$$

then $\partial \Omega \in B_{p}^{l-1 / p}$.

Note that the convergence requirement (3.7), the most restrictive assumption on $\Omega$ made in Corollary 3 , is in a sense a sharp condition for solvability of the Dirichlet problems (3.17) and (3.18) in $W_{p}^{l}(\Omega)$. The corresponding domain is constructed with the help of the same function $\omega$ as in the case of the Neumann problem (3.12) (see Example 15.6.1 in [57]). 
Example 15.5.2 from [57] shows that surfaces in the class $M_{p}^{l-1 / p}(\delta)$ with $p(l-1)<$ $n$ may have

$$
\text { conic vertices if } n>p(l-1)
$$

and

$$
s \text {-dimensional edges if } s<n-p(l-1) \text {. }
$$

Suppose that for any point $O \in \partial \Omega$ there exists a neighborhood $U$ such that $U \cap \Omega$ is $C^{\infty}$-diffeomorphic to the domain

$$
\mathbb{R}^{s} \times\left\{(x, y): y>\varphi\left(x_{s+1}, \ldots, x_{n-1}\right)\right\}, \quad 0 \leq s \leq n-2,
$$

i.e., the dimensions of boundary singularities are at most $n-1-s$. Then (3.4) is equivalent to

$$
\|\nabla \varphi\|_{M B_{p}^{l-1-1 / p}\left(\mathbb{R}^{n-1-s}\right)} \leq c \delta
$$

and, in particular, it takes the form

$$
\|\nabla \varphi\|_{B_{p, \text { unif }}^{l-1-1 / p}\left(\mathbb{R}^{n-1-s}\right)} \leq c \delta
$$

if $n-s<p(l-1) \leq n$. In two last inequalities $c$ is a positive constant depending on $p, n, l$, and $s$. In other words, $\partial \Omega \in M_{p}^{l-1 / p}(\delta)$ if and only if the $(n-1-s)$ dimensional domain $\left\{(x, y): y>\varphi\left(x_{s+1}, \ldots, x_{n-1}\right)\right\}$ belongs to $M_{p}^{l-1 / p}(c \delta)$. Here $\left.B_{p, \text { unif }}^{l-1-1 / p}\left(\mathbb{R}^{n-1-s}\right)\right)$ is the space of functions with the norm

$$
\sup _{x \in \mathbb{R}^{n-1-s}}\left(\left(\int_{\mathcal{B}_{1}}\left\|\Delta_{h} \nabla_{l-2} u\right\|_{L_{p}\left(\mathcal{B}_{1}(x)\right)}^{p}|h|^{2+s-n-p} d h\right)^{1 / p}+\|u\|_{W_{p}^{l-2}\left(\mathcal{B}_{1}(x)\right)}\right)
$$

and $\mathcal{B}_{1}(x)$ is the ball in $\mathbb{R}^{n-1-s}$ of unit radius and center at $x, \mathcal{B}_{1}=\mathcal{B}_{1}(0)$.

\subsection{Dirichlet problem in a variational formulation}

It turns out that for equations and systems of order higher than two, the formulation of the Dirichlet problem can be changed so that the solvability condition $\Omega \in M_{p}^{l-1 / p}(\delta)$ is replaced by the better one $\Omega \in M_{p}^{l+1-m-1 / p}(\delta)$. We comment on this referring to Section 14.5 of [57].

Let $\Omega$ be open subset $\mathbb{R}^{n}$ and let $P$ be the operator (3.13), where $A_{\alpha \beta} \in C^{l-m}(\bar{\Omega})$, $l \geq m$. Further, let the coercivity condition (3.14) hold for $u \in C_{0}^{\infty}(\Omega)$. 
We say that $u \in W_{p}^{l}(\Omega)$ is a variational solution of the Dirichlet problem if

$$
P u=f, \quad u-g \in W_{p}^{l}(\Omega) \cap \stackrel{\circ}{W}_{p}^{m}(\Omega),
$$

where $f$ and $g$ are given functions in the spaces $W_{p}^{l-2 m}(\Omega)$ and $W_{p}^{l}(\Omega)$ respectively.

We present an a priori estimate for solutions of problem (3.19).

\section{Theorem 11 If}

(i) either $p(l-m) \leq n, 1<p<\infty$ and $\partial \Omega \in M_{p}^{l+1-m-1 / p}(\delta)$, where $\delta$ depends on the coefficients of the principal homogeneous part of $P$ or

(ii) $p(l-m)>n, 1<p<\infty$, and $\partial \Omega \in B_{p}^{l+1-m-1 / p}$,

then

$$
\|u\|_{W_{p}^{l}(\Omega)} \leq c\left(\|P u\|_{W_{p}^{l-2 m}(\Omega)}+\|u\|_{L_{1}(\Omega)}\right)
$$

for all $u \in\left(W_{p}^{l} \cap \stackrel{\circ}{W}_{p}^{m}\right)(\Omega)$.

Next we state two corollaries of (3.20).

Proposition 4 Let $\Omega$ satisfy the conditions of Theorem 11.

(i) If the kernel of the operator

$$
P:\left(W_{p}^{l} \cap \stackrel{\circ}{W}_{p}^{m}\right)(\Omega) \rightarrow W_{p}^{l-2 m}(\Omega)
$$

is trivial, then the norm $\|u\|_{L_{1}(\Omega)}$ in (3.20) can be omitted.

(ii) The kernel of the operator (3.21) is finite-dimensional and the range of this operator is closed.

Proposition 5 Let $\Omega$ satisfy the conditions of Theorem 11. Further, let $U$ and $V$ be open bounded subsets of $\mathbb{R}^{n}, \bar{U} \subset V$ and $u \in\left(W_{p}^{l} \cap \stackrel{\circ}{W}_{p}^{m}\right)(\Omega)$. Then

$$
\|u\|_{W_{p}^{l}(U \cap \Omega)} \leq c\left(\|P u\|_{W_{p}^{l-2 m}(V \cap \Omega)}+\|u\|_{L_{1}(V \cap \Omega)}\right) .
$$

Finally we formulate a theorem concerning the solvability of (3.19) established in [57].

Let the coercivity condition (3.14) hold for all $u \in C_{0}^{\infty}(\Omega)$. Then, as is well known, the equation $P u=f$ with $f \in W_{2}^{-m}(\Omega)$ is uniquely solvable in $\stackrel{\circ}{W}_{2}^{m}(\Omega)$.

Theorem 12 Let $\partial \Omega \in M_{p}^{l+1-m-1 / p}$ for $p(l-m) \leq n$ and let $\partial \Omega$ belong to the class $B_{p}^{l+1-m-1 / p}$ for $p(l-m)>n$.

(i) If $f \in W_{p}^{l-2 m}(\Omega) \cap W_{2}^{-m}(\Omega), g \in W_{p}^{l}(\Omega) \cap W_{2}^{m}(\Omega), 1<p<\infty$, and if $u \in W_{2}^{m}(\Omega)$ is such that $P u=f, u-g \in \stackrel{\circ}{W}_{2}^{m}(\Omega)$, then $u \in W_{p}^{l}(\Omega)$ and $u-g \in \stackrel{\circ}{W}_{p}^{m}(\Omega)$.

(ii) The problem (3.19) has exactly one solution $u \in W_{p}^{l}(\Omega)$. 
3.6 Strong solvability of the Dirichlet problem for the Stokes system

Here we complement Sect. 2 by a theorem on solvability of the Dirichlet problem for the Stokes system in weighted Sobolev spaces of higher order.

Let $l$ be noninteger, $l>1$. We use the notation $M_{p}^{l}(\delta)$ for the class of threedimensional Lipschitz graph domains subject to

$$
\|\nabla \varphi\|_{M B_{p}^{l-1}\left(\mathbb{R}^{2}\right)} \leq \delta
$$

for an arbitrary coordinate system on $\partial \Omega$, where $\delta$ is a positive number.

We conclude this section by stating a strong solvability result for the Dirichlet problem for the Stokes system

$$
\begin{aligned}
\Delta u-\nabla \pi & =f, \quad \operatorname{div} u=g \quad \text { in } \Omega, \\
\operatorname{Tr} u=h & \text { on } \partial \Omega .
\end{aligned}
$$

We assume that $g$ and $h$ satisfy the compatibility condition (2.3) and use the space $W_{p}^{m, a}$ introduced in Sect. 1.3. The proof is essentially the same as that of Theorem 15.1.2 in [57].

Theorem 13 Let $p \in(1, \infty), a=1-\{l\}-1 / p$, where $l$ is noninteger, $l>1$. Suppose that $\partial \Omega \in B_{p}^{l}$ for $p(l-1)>2$ and $\partial \Omega \in M_{p}^{l}(\delta)$ with some $\delta=\delta(p, l)$ for $p(l-1) \leq 2$. Then, for every triple

$$
(f, g, h) \in W_{p}^{[l]-1, a}(\Omega) \times W_{p}^{[l], a}(\Omega) \times B_{p}^{l}(\partial \Omega)
$$

there exists a unique solution $(u, \pi)$ of the problem $(3.22)$ in $W_{p}^{[l]+1, a}(\Omega) \times W_{p}^{[l], a}(\Omega)$.

Note that under the conditions of the last theorem, the operator

$$
\begin{aligned}
& W_{p}^{[l]+1, a}(\Omega) \times W_{p}^{[l], a}(\Omega) \ni(u, \pi) \\
& \Longrightarrow(\Delta u-\nabla \pi, \operatorname{div} u, \operatorname{Tr} u) \in W_{p}^{[l]-1, a}(\Omega) \times W_{p}^{[l], a}(\Omega) \times B_{p}^{l}(\partial \Omega)
\end{aligned}
$$

is continuous.

\section{Asymptotic behavior of solutions to elliptic equations near a point}

\subsection{Asymptotics of solutions near Lipschitz boundary}

Results of a new type were obtained by Kozlov and Maz'ya [44] for solutions of the Dirichlet problem for higher order elliptic equations in Lipschitz graph domains. We mean an explicit description of the asymptotic behaviour of solutions near a point $\mathcal{O}$ of the Lipschitz boundary.

Consider the special Lipschitz graph domain

$$
G=\left\{x=\left(x^{\prime}, x_{n}\right) \in \mathbb{R}^{n}: x_{n}>\varphi\left(x^{\prime}\right)\right\},
$$


where $\varphi(0)=0$ and $\varphi$ has a small Lipschitz constant. The authors of [44] study solutions of arbitrary strongly elliptic equation of order $2 m$ with constant complex-valued coefficients

$$
L\left(\partial_{x}\right) u(x)=f(x) \quad \text { on } B_{1} \cap G
$$

with zero Dirichlet data on $\left(B_{1} \cap \partial G\right) \backslash \mathcal{O}$, where $B_{\rho}=\{x:|x|<\rho\}$ and $\partial_{x}=$ $\left(\partial_{1}, \ldots, \partial_{n}\right)$. It is assumed that the operator $L$ has no lower-order terms and the coefficient in front of $\partial_{n}^{2 m}$ is equal to $(-1)^{m}$.

One of the results in [44] is the existence of a solution $\mathcal{U} \in W_{2}^{m}(G)$ of the homogeneous equation (4.1) which admits the asymptotic representation

$$
\begin{aligned}
\mathcal{U}(x)= & \exp \left(-\int_{|x|<\left|y^{\prime}\right|<1 / 2} \varphi\left(y^{\prime}\right) \partial_{n}^{m} E\left(y^{\prime}, 0\right) d y^{\prime}+O\left(\int_{|x|}^{1} \varkappa^{2}(\rho) \frac{d \rho}{\rho}\right)\right) \\
& \times\left(\left(x_{n}-\varphi\left(x^{\prime}\right)\right)^{m}+O\left(|x|^{m+1-\varepsilon}\left(\int_{|x|}^{1} \varkappa(\rho) \frac{d \rho}{\rho^{2-\varepsilon}}+1\right)\right)\right)
\end{aligned}
$$

Here $\varepsilon$ is a poisitive constant,

$$
\varkappa(\rho)=\sup _{\left|y^{\prime}\right|<\rho}\left|\nabla \varphi\left(y^{\prime}\right)\right|
$$

and $E$ is the Poisson solution of the equation $L\left(\partial_{x}\right) E(x)=0$ in the upper half-space $\mathbb{R}_{+}^{n}$ which is positive homogeneous of degree $m-n$ and is subject to the Dirichlet conditions on the hyperplane $x_{n}=0$

$$
\partial_{n}^{j} E=0, \quad 0 \leq j \leq m-2, \quad \text { and } \quad \partial_{n}^{m-1} E=\delta\left(x^{\prime}\right),
$$

where $\delta$ is the Dirac function.

It is also shown that a multiple of $\mathcal{U}$ is the main term in the asymptotic representation of an arbitrary solution $u$ if both $u$ and $f$ are subject to mild growth conditions near $\mathcal{O}$.

Solutions with the weakest possible singularity at $\mathcal{O}$ are studied in [44] as well. The authors construct a solution $\mathcal{U}$ of the homogeneous equation (4.1) which is subject to the asymptotic formula

$$
\begin{aligned}
\mathcal{U}(x)= & \exp \left(\int_{|x|<\left|y^{\prime}\right|<1 / 2} \varphi\left(y^{\prime}\right) \partial_{n}^{m} E\left(y^{\prime}, 0\right) d y^{\prime}+O\left(\int_{|x|}^{1} x^{2}(\rho) \frac{d \rho}{\rho}\right)\right) \\
& \times\left(E\left(x_{n}-\varphi\left(x^{\prime}\right)\right)+O\left(|x|^{m-n+1-\varepsilon}\left(\int_{|x|}^{1} \varkappa(\rho) \frac{d \rho}{\rho^{2-\varepsilon}}+1\right)\right) .\right.
\end{aligned}
$$


The asymptotic formulas (4.2) and (4.3) can be simplified under additional conditions on $\varkappa(\rho)$. Let, in particular,

$$
\int_{0}^{1} x^{2}(\rho) \frac{d \rho}{\rho}<\infty .
$$

Then, in the special case of the polyharmonic equation

$$
(-\Delta)^{m} u=0 \text { on } B_{1} \cap G,
$$

any solution $u$ satisfying $|u(x)|=O\left(|x|^{m-n+1-\varepsilon}\right)$ is subject to the following alternatives: either

$$
u(x) \sim C \frac{\left(x_{n}-\varphi\left(x^{\prime}\right)\right)^{m}}{|x|^{n}} \exp \left(m \frac{\Gamma(n / 2)}{\pi^{n / 2}} \int_{|x|<\left|y^{\prime}\right|<1 / 2} \varphi\left(y^{\prime}\right) \frac{d y^{\prime}}{\left|y^{\prime}\right|^{n}}\right)
$$

or

$$
u(x) \sim C\left(x_{n}-\varphi\left(x^{\prime}\right)\right)^{m} \exp \left(-m \frac{\Gamma(n / 2)}{\pi^{n / 2}} \int_{|x|<\left|y^{\prime}\right|<1 / 2} \varphi\left(y^{\prime}\right) \frac{d y^{\prime}}{\left|y^{\prime}\right|^{n}}\right)
$$

\subsection{Asymptotics of solutions to equations with discontinuous coefficients near a smooth boundary}

Proofs of the just mentioned results in [44] rely upon the papers [42,43] on the asymptotic formulas for solutions to the Dirichlet problem for arbitrary even order $2 m$ strongly elliptic equations of divergence form near a point $\mathcal{O}$ at the smooth boundary. It is required only that the coefficients of the principal part of the operator have small oscillation near this point, and the coefficients in lower order terms are allowed to have singularities at the boundary.

We say a few words on the proof of asymptotic formulas in $[42,43]$. The elliptic equation is transformed to a first-order evolution system with the matrix whose entries are partial differential operators on the hemisphere with time dependent coefficients. Thus, the question of asymptotics of solutions to the original Dirichlet problem is reduced to the study of the long-time behaviour of solutions of the evolution system just mentioned. The structure of the operator matrix in the system is rather complicated, because it has been obtained from a higher order partial differential equation in the variational form. Moreover, the study of this system is aggreviated by the scantiness of information about the behaviour of the operator matrics at infinity. This difficulty is overcome by a right choice of function spaces, characterizing the solutions and the right-hand side of the evolution system by certain seminorms depending on time. To obtain an asymptotic formula for the solution, the authors apply a particular spectral splitting of the system into one-dimensional and infinite-dimensional parts. A general 
asymptotic theory of differential equations with operator coefficients in Banach spaces which is the basis of [42,43] is developed in [41].

As an illustration, we describe a corollary of the main result in [42] concerning second order equations. Consider the uniformly elliptic equation

$$
-\operatorname{div}(A(x) \nabla u(x))=f(x) \text { in } \Omega
$$

complemented by the Dirichlet condition

$$
u=0 \text { on } \partial \Omega
$$

where $\Omega$ is a domain in $\mathbb{R}^{n}$ with smooth boundary. We assume that elements of the $n \times n$ matrix $A(x)$ are measurable and bounded complex-valued functions. One consideres a solution $u$ with a finite Dirichlet integral and supposes, for simplicity, that $f=0$ in a certain $\delta$-neighborhood $\Omega_{\delta}=\{x \in \Omega:|x|<\delta\}$ of the origin. Further, it is assumed that there exists a constant symmetric matrix $A$ with positive definite real part such that the function

$$
\sigma(r):=\sup _{\Omega_{r}}\|A(x)-A\|
$$

does not exceed a sufficiently small constant for $r<\delta$.

We introduce the function

$$
\mathcal{R}(x)=\frac{\langle(A(x)-A) v, v\rangle-n\left\langle A^{-1}(A(x)-A) v, x\right\rangle\langle v, x\rangle\left\langle A^{-1} x, x\right\rangle^{-1}}{\left|S^{n-1}\right|(\operatorname{det} A)^{1 / 2}\left\langle A^{-1} x, x\right\rangle^{n / 2}},
$$

where $\left|S^{n-1}\right|$ is the Lebesgue measure of the unit sphere in $\mathbb{R}^{n},\langle z, \zeta\rangle=z_{1} \overline{\zeta_{1}}+\cdots+$ $z_{n} \overline{\zeta_{n}}$ and $v$ is the interior unit normal at a point $\mathcal{O}$ on the boundary of $\Omega$. (For the notation $(\operatorname{det} A)^{1 / 2}$ see [35], Sect. 3.4).

The following asymptotic formula for an arbitrary solution of (4.4), (4.5) with finite energy integral is a special case of the main theorem in [42]:

$$
\begin{aligned}
u(x)= & C \exp \left(-\int_{\Omega_{\delta} \backslash \Omega_{|x|}} \mathcal{R}(y) d y+O\left(\int_{|x|}^{\delta} \sigma(\rho)^{2} \frac{d \rho}{\rho}\right)\right) \\
& \times\left(\operatorname{dist}(x, \partial \Omega)+O\left(|x|^{2-\varepsilon} \int_{|x|}^{\delta} \sigma(\rho) \frac{d \rho}{\rho^{2-\varepsilon}}\right)\right)+O\left(|x|^{2-\varepsilon}\right),
\end{aligned}
$$

where $C=$ const and $\varepsilon$ is a small positive number.

Using (4.6), it is an easy matter to derive sharp two-sided estimate for the Hölder exponent of $u$ at the origin. Another direct application of (4.6) is the following criterion. 
Under the condition

$$
\int_{0}^{\delta} \sigma(\rho)^{2} \frac{d \rho}{\rho}<\infty
$$

all solutions $u$ are Lipschitz at the origin if and only if

$$
\liminf _{r \rightarrow+0} \int_{\Omega_{\delta} \backslash \Omega_{r}} \operatorname{Re} \mathcal{R} d x>-\infty .
$$

Needless to say, this new one-sided restriction (4.8) is weaker than the classical Dini condition at the origin. The complementary assumption (4.7) appeared previously in several papers dealing with other problems of the boundary behaviour of solutions to equation (4.4) (see the articles by Fabes et al. [27], by Dahlberg [17], by Kenig [39]).

Let $v$ be a solution of the equation (4.4) complemented by the Dirichlet condition

$$
v=0 \quad \text { on } \partial \Omega \backslash\{\mathcal{O}\}, \mathcal{O} \in \partial \Omega,
$$

which has an infinite energy integral and the least possible singularity. We state a particular case of the main theorem in [42] which is the following asymptotic representation for $v$ :

$$
\begin{aligned}
v(x)= & C \exp \left(\int_{\Omega_{\delta} \backslash \Omega_{|x|}} \mathcal{R}(y) d y+O\left(\int_{|x|}^{\delta} \sigma(\rho)^{2} \frac{d \rho}{\rho}\right)\right) \\
& \times\left(\frac{\operatorname{dist}(x, \partial \Omega)}{\left\langle A^{-1} x, x\right\rangle^{n / 2}}+O\left(|x|^{2-n-\varepsilon} \int_{|x|}^{\delta} \sigma(\rho) \frac{d \rho}{\rho^{2-\varepsilon}}\right)\right)+O\left(|x|^{1-\varepsilon}\right),
\end{aligned}
$$

where $C=$ const and $\varepsilon$ is a small positive number.

In general, theorems proved in $[42,43]$ provide asymptotic formulas similar to (4.6) and (4.9) for solutions of the Dirichlet problem for the higher order uniformly elliptic equations with complex-valued coefficients

$$
\sum_{0 \leq|\alpha|,|\beta| \leq m}\left(-\partial_{x}\right)^{\alpha}\left(\mathcal{L}_{\alpha \beta}(x) \partial_{x}^{\beta} u(x)\right)=f(x) \text { on } B_{\delta}^{+},
$$

where $B_{\delta}^{+}=\mathbb{R}_{+}^{n} \cap B_{\delta}, \mathbb{R}_{+}^{n}=\left\{x=\left(x^{\prime}, x_{n}\right) \in \mathbb{R}^{n}: x_{n}>0\right\}$ and $B_{\delta}=\left\{x \in \mathbb{R}^{n}\right.$ : $|x|<\delta\}$. The only a priori assumption on the coefficients $\mathcal{L}_{\alpha \beta}$ is smallness of the function

$$
\sum_{|\alpha|=|\beta|=m}\left|\mathcal{L}_{\alpha \beta}(x)-L_{\alpha \beta}\right|+\sum_{|\alpha+\beta|<2 m} x_{n}^{2 m-|\alpha+\beta|}\left|\mathcal{L}_{\alpha \beta}(x)\right|,
$$

where $x \in B_{\delta}^{+}$and $L_{\alpha \beta}$ are constants. 
4.3 Corollaries of the asymptotic formulas in Sect. 4.1

The last section in [44] concerns, in particular, solutions to the Dirichlet problem for elliptic equations of order $2 m$ with constant coefficients in plane domains with a small Lipschitz constant of the boundary as well as arbitrary bounded plane convex domans $\Omega$. Let $\Omega$ be a bounded domain in $\mathbb{R}^{2}$. Consider a strongly elliptic operator with constant coefficients

$$
L\left(\partial_{x}\right)=\sum_{0 \leq k \leq 2 m} a_{k} \partial_{1}^{k} \partial_{2}^{2 m-k},
$$

and denote by $w$ a weak solution to the Dirichlet problem

$$
L\left(\partial_{x}\right) w=f, \quad w \in \stackrel{\circ}{W}_{2}^{m}(\Omega)
$$

If $f \in W_{2}^{-m}(\Omega)$, this problem is uniquely solvable. let us assume that

$$
\Omega \cap B_{2 \delta_{0}}=\left\{\left(x_{1}, x_{2}\right): x_{2}>\varphi\left(x_{1}\right),|x|<2 \delta_{0}\right\},
$$

where $\varphi$ is a Lipschitz function on $\left[-2 \delta_{0},-2 \delta_{0}\right]$ and $\varphi(0)=0$. Note that one does not require convexity of $\varphi$.

The next result concerning solutions to problem (4.10), which stems from (4.2), is as follows.

Theorem 14 Suppose that the Lipschitz norm of $\varphi$ on $\left[-2 \delta_{0},-2 \delta_{0}\right]$ does not exceed a certain constant depending on the coefficients of L. Let $f$ be equal to zero in $\Omega \cap B_{2 \delta}$. Then, there exist positive constants $b$ and $c$ depending only on $m$ and the coefficients of $L$ such that for all $\delta \in\left(0, \delta_{0}\right), x \in \Omega \cap B_{\delta}$ and $k=1, \ldots, m-1$,

$$
\begin{aligned}
\left|\nabla_{k} w(x)\right| \leq & c A(2 \delta)|x|^{m-k} \\
& \times \exp \left(-a \int_{|x|}^{\delta} \frac{\varphi(\rho)-\varphi(-\rho)}{\rho^{2}} d \rho+b \int_{|x|}^{\delta} \max _{|t|<\rho}\left|\varphi^{\prime}(t)\right|^{2} \frac{d \rho}{\rho}\right) .
\end{aligned}
$$

Here

$$
A(\delta)=\delta^{-1-m}\|w\|_{L_{2}\left(\Omega \cap B_{\delta}\right)}
$$

and the notation

$$
a=\frac{1}{2 \pi} \operatorname{Im} \sum_{1 \leq k \leq m}\left(\zeta_{k}^{+}-\zeta_{k}^{-}\right)
$$

is used, where $\zeta_{1}^{+}, \ldots, \zeta_{m}^{+}$and $\zeta_{1}^{-}, \ldots, \zeta_{m}^{-}$are roots of the polynomial $L(1, \zeta)$ with positive and negative imaginary parts, respectively. This value of a is best possible. 
Note that for the operator $\Delta^{m}$ one has $\zeta_{k}^{ \pm}= \pm i$, which implies $a=-m / \pi$.

The next assertion is a consequence of Theorem 14 when the function $\varphi$ is convex.

Theorem 15 Suppose that the function $\varphi$ describing the domain $\Omega$ near the point $\mathcal{O}$ is non-negative and convex, and $\left|\varphi^{\prime}( \pm 2 \delta)\right|$ does not exceed a sufficiently small constant $l_{0}$ depending on $m$ and the coefficients of $L\left(\partial_{x}\right)$. Furthermore, let $f$ be zero in $\Omega \cap B_{2 \delta}$ and let $w$ be a solution of (4.10), which is extended by zero outside $\Omega$. Then

$$
\left\|\nabla_{m} w\right\|_{L_{\infty}\left(B_{\delta}\right)} \leq c A(8 \delta)
$$

where $\delta<\delta_{0} / 8$ and $A(\delta)$ is given by (4.12).

One of the main results obtained in [44] concerns the Green function $G_{L}$ of the Dirichlet problem for the operator $L$ with real coefficients.

Theorem 16 Let $\Omega$ be an arbitrary bounded convex domain in $\mathbb{R}^{2}$ and let the coefficients of $L$ be real. Then, for all $x, y$ in $\Omega$,

$$
\sum_{|\alpha|=|\beta|=m}\left|\partial_{x}^{\alpha} \partial_{y}^{\beta} G_{L}(x, y)\right| \leq C|x-y|^{-2},
$$

where $C$ is a positive constant depending on $\Omega$.

The case of complex coefficients is more complicated.

Theorem 17 Let $L$ be an arbitrary strongly elliptic operator with complex coefficients. Suppose that $\Omega$ is a bounded convex domain such that the jumps of all angles between the exterior normal vector to $\partial \Omega$ and the $x$-axis is smaller than a constant depending on $m$ and the coefficients of $L\left(\partial_{x}\right)$. Then, for all $x, y$ in $\Omega$, estimate (4.14) holds.

Theorem 16 implies the following regularity result.

Corollary 5 Let $\Omega$ be an arbitrary bounded convex domain in $\mathbb{R}^{2}$ and let the coefficients of $L$ be real. Then the solution $w$ of problem (4.10) with $f \in W_{q}^{1-m}(\Omega), q>2$, satisfies

$$
\sum_{|\alpha| \leq m}\left\|D^{\alpha} w\right\|_{L_{\infty}(\Omega)} \leq C\|f\|_{W_{q}^{1-m}(\Omega)},
$$

where the constant $C$ depends on $\Omega, m, q$, and the coefficients of $L\left(\partial_{x}\right)$.

Generally, this assertion does not hold for operators with complex coefficients. More precisely, if there exists an angle vertex on the boundary of a convex domain $\Omega$, one can construct a second order strongly elliptic operator $L\left(\partial_{x}\right)$ with complex coefficients such that the Dirichlet problem (4.10) with $f \in C(\bar{\Omega})$ has a solution with unbounded gradient (see [45], Sect. 8.4.3). By Theorem 17, the statement of Corollary 5 for $L$ with complex coefficients holds if jumps of the normal vector are either absent or small. 
4.4 Classical asymptotics of solutions near a point of the domain

Now, we present some results borrowed from Sect. 14.9 of the book by Kozlov and Maz'ya [41] which concerns the asymptotic behaviour of solutions to elliptic equations near an interior point $O$ of the domain. Here a modified Dini-type condition on the coefficients is introduced which guarantees the preservation of the asymptotics of solutions to the main part of the equation with coefficients frozen at $O$.

Let

$$
P\left(D_{x}\right)=\sum_{|\alpha|=2 m} p_{\alpha} D_{x}^{\alpha}
$$

and let $G$ denote the Green matrix of this operator, i.e. the solution of the system

$$
P\left(D_{x}\right) G(x)=I_{l} \delta(x) \text { in } \mathbb{R}^{n},
$$

where $I_{l}$ is the $l \times l$ identity matrix and $\delta$ is the Dirac function. It is well-known (see John's book [37]) that $G$ admits the representation

$$
G(x)= \begin{cases}l l r^{2 m-n} Q(\omega) & \text { if } 2 m \geq n, \quad n \text { odd, or } 2 m<n, \\ R(x) \log r+r^{2 m-n} S(\omega) & \text { if } 2 m \geq n, n \text { even, }\end{cases}
$$

where $Q$ and $S$ are smooth matrix-functions on the unit sphere in $\mathbb{R}^{n}$ and $R$ is a homogeneous polynomial matrix of degree $2 m-n$.

Let us consider the elliptic operator

$$
Q\left(x, D_{x}\right)=\sum_{|\alpha| \leq 2 m} q_{\alpha}(x) D_{x}^{\alpha}
$$

with measurable coefficients in the punctured ball $B_{r_{0}} \backslash\{0\}$. We introduce the function

$$
S(r)=\sup _{K_{r}}\left\{\sum_{|\alpha|=2 m}\left|q_{\alpha}(x)-p_{\alpha}\right|+\sum_{|\alpha|<2 m}|x|^{2 m-|\alpha|}\left|q_{\alpha}(x)\right|\right\}
$$

where $K_{r}=\left\{x \in \mathbb{R}^{n}: e^{-1} r<|x|<r\right\}$, and assume that $S(r)$ does not exceed a small positive constant. We shall also use the notation

$$
\|u\|_{\mathcal{W}_{2}^{2 m}\left(K_{r}\right)}=\left(\sum_{|\alpha| \leq 2 m} r^{2|\alpha|-n}\left\|D_{x}^{\alpha} u\right\|_{L_{2}\left(K_{r}\right)}^{2}\right)^{1 / 2}
$$

We formulate three theorems on the asymptotic representation as $x \rightarrow 0$ for solutions $u \in W_{2, l o c}^{2 m}\left(B_{r_{0}} \backslash\{0\}\right)$ of

$$
Q\left(x, D_{x}\right) u=0 \quad \text { on } B_{r_{0}} \backslash\{0\}
$$


satisfying, for small $r$,

$$
\|u\|_{\mathcal{W}_{2}^{2 m}\left(K_{r}\right)}=O\left(r^{k+\delta}\right)
$$

with some $\delta>0$ and integer $k$.

Theorem 18 (see Theorem 14.9.1 in [41]) Let $2 m<n$ and

$$
\int_{0}^{r_{0}} S(r)|\log r|^{\gamma-1} \frac{d r}{r}<\infty
$$

where $\gamma$ is a positive integer.

(i) If $k \geq 0$, then

$$
u(x)=\sum_{|\alpha|=k+1} c_{\alpha} x^{\alpha}+v(x),
$$

where $c_{\alpha}=$ const and

$$
\|v\|_{\mathcal{W}_{2}^{2 m}\left(K_{r}\right)}=o\left(r^{k+1}|\log r|^{1-\gamma}\right)
$$

as $r \rightarrow 0$.

(ii) If $k \leq 2 m-n-1$, then

$$
u(x)=\sum_{|\alpha|=2 m-n-k-1} C_{\alpha} D_{x}^{\alpha} G(x)+v(x),
$$

where $C_{\alpha}=$ const and $G$ is the Green matrix introduced by (4.16) and $v$ satisfies (4.21).

(iii) If $k=2 m-n$, then

$$
u(x)=\text { const }+v(x),
$$

where

$$
\|v\|_{\mathcal{W}_{2}^{2 m}\left(K_{r}\right)}=o\left(|\log r|^{1-\gamma}\right)
$$

as $r \rightarrow 0$.

The asymptotics (4.23) can be made more precise under the assumption that the operator $Q$ contains no derivatives of order $|\alpha|<s$, that is

$$
Q\left(x, D_{x}\right)=\sum_{s \leq|\alpha| \leq 2 m} q_{\alpha}(x) D_{x}^{\alpha} .
$$


The formula (4.23) can be replaced by

$$
u(x)=\sum_{|\alpha| \leq s} c_{\alpha} x^{\alpha}+v(x)
$$

where

$$
\|v\|_{\mathcal{W}_{2}^{2 m}\left(K_{r}\right)}=o\left(r^{s}|\log r|^{1-\gamma}\right)
$$

as $r \rightarrow 0$.

Theorem 19 (see Theorem 14.9.2 in [41]) Let $n$ be odd, $2 m>n$ and let $S$ be subject to (4.19). Then

$$
u(x)=\sum_{|\alpha|=k+1} c_{\alpha} x^{\alpha}+\sum_{|\beta|=2 m-n-k-1} C_{\beta} D_{x}^{\beta} G(x)+v(x),
$$

where $c_{\alpha}$ and $C_{\beta}$ are constants and $v$ satisfies (4.21). If either $k<-1$ or $k>2 m-n$, then the first or the second sum in (4.24) should be omitted.

Theorem 20 (see Theorem 14.9.4 in [41]) Let $n$ be even, $2 m>n$.

(i) If $k \leq-2$ and (4.19) holds, then $u$ satisfies (4.14) with $v$ subject to (4.21).

(ii) If $k \geq 2 m-n$ and (4.19) holds, then $u$ satisfies (4.20) with $v$ subject to (4.21).

(iii) Let $-1 \leq k \leq 2 m-n-1$ and let (4.19) be valid with $\gamma \geq 2$. Then $u$ is represented by (4.24) with

$$
\|u\|_{\mathcal{W}_{2}^{2 m}\left(K_{r}\right)}=o\left(r^{k+1}|\log r|^{3-\gamma}\right)
$$

as $r \rightarrow 0$.

4.5 Asymptotics of solutions of the second order equation with square-Dini coefficients

The asymptotic behaviour of solutions near the isolated point $O$ of the domain was recently considered by Maz'ya and McOwen $[49,50]$ for the case of the second order elliptic operator in nondevergence form

$$
\mathcal{L}\left(x, D_{x}\right) u(x)=\sum_{1 \leq i, j \leq n} a_{i j}(x) \partial_{i} \partial_{j} u
$$

It is assumed that the coefficients have the modulus of continuity $\omega$ satisfying the square-Dini condition

$$
\int_{0}^{1} \omega^{2}(t) \frac{d t}{t}<\infty
$$


If the coefficients are real, then, without loss of generality one can put $a_{i j}(0)=\delta_{i j}$.

An important role is played by the function

$$
I(r)=\frac{1}{\left|S^{n-1}\right|} \int_{r<|z|<\varepsilon}\left(\operatorname{trace}\left(\mathbf{A}_{z}\right)-n \frac{\left\langle\mathbf{A}_{z} z, z\right\rangle}{|z|^{2}}\right) \frac{d z}{|z|^{n}},
$$

where $\mathbf{A}_{z}$ stands for the matrix $a_{i j}(z),\langle$,$\rangle is the inner product in \mathbb{R}^{n}$, and $\varepsilon$ is a sufficiently small positive number.

If the coefficients are subject to the usual Dini condition

$$
\int_{0}^{1} \omega(t) \frac{d t}{t}<\infty
$$

then, obviously, there exists a finite limit of $I(r)$ as $r \rightarrow 0$, but (4.28) is not necessary for the existence of this limit. In general, under (4.26), $I(r)$ may be unbounded as $r \rightarrow 0$, but, clearly, for every $\lambda>0$ there exists $C_{\lambda}$ such that

$$
|I(r)| \leq \lambda|\log r|+C_{\lambda} \quad \text { for } 0<r<\varepsilon .
$$

The results in [49] and [50] are formulated in terms of the $L_{p}$-means

$$
M_{p}(w, r):=\left(f_{r<|x|<2 r}|w(x)|^{p} d x\right)^{1 / p} .
$$

The main theorem in [49] can be stated as follows.

\section{Theorem 21 Suppose that}

$$
\left|a_{i j}(x)-\delta_{i j}\right| \leq \omega(|x|) \text { as } x \rightarrow O,
$$

where $\omega$ satisfies (4.26). For $p \in(1, \infty)$ and $\varepsilon>0$ sufficiently small, there exists $a$ weak solution $Z \in L_{p, l o c}\left(B_{\varepsilon}\right)$ of

$$
\sum_{1 \leq i, j \leq n} \partial_{i} \partial_{j}\left(a_{i j}(x) Z\right)=0
$$

satisfying

$$
Z(x)=e^{-I(|x|)}(1+\zeta(x))
$$

where the remainder term $\zeta$ satisfies

$$
M_{p}(\zeta, r) \leq c \max (\omega(r), \sigma(r)) \text { with } \sigma(r):=\int_{0}^{r} \frac{\omega^{2}(t)}{t} d t
$$


Moreover, if $u \in L_{p, l o c}\left(\overline{B_{\varepsilon}} \backslash\{O\}\right)$ is a weak solution of

$$
\sum_{1 \leq i, j \leq n} \partial_{i} \partial_{j}\left(a_{i j}(x) u\right)=0
$$

in $B_{\varepsilon}$ subject to the growth condition

$$
M_{p}(u, r) \leq c r^{2-n+\varepsilon_{0}},
$$

for some $\varepsilon_{0}>0$, then there exists a constant $C$, depending on $u$, such that

$$
u(x)=C Z(x)+w(x)
$$

where the remainder term $w$ satisfies

$$
M_{p}(w, r) \leq c r^{1-\varepsilon_{1}}
$$

for $0<r<\varepsilon$ and any $\varepsilon_{1}>0$.

Combining (4.34) and (4.32), one obtains the asymptotic representation

$$
u(x)=c e^{-I(|x|)}|x|^{n}(1+o(1)) \quad \text { as } x \rightarrow O .
$$

Now let us turn to the behaviour of a solution to the homogeneous nondivergence equation with an isolated singularity at $x=O$. We assume that $n>2$. The results obtained in [50] imply the existence of the solution

$$
Z(x) \sim \frac{|x|^{2-n}}{n-2} e^{I(|x|)} \quad \text { as } x \rightarrow O .
$$

The behaviour of $I(|x|)$ as $x \rightarrow O$ not only controls the leading asymptotics of $Z(x)$ but whether we can solve the equation

$$
\mathcal{L}\left(x, D_{x}\right) F(x)=\delta(x)
$$

There are three important cases to consider.

1. $I(0)=\lim _{x \rightarrow O} I(|x|)$ exists and is finite.

In this case $Z(x)$ may be scaled by a constant factor to make it asymptotic to the fundamental solution for the Laplacian. In fact, the distributional equation

$$
-\mathcal{L}\left(x, D_{x}\right) Z(x)=C \delta(x)
$$

can be solved to find

$$
C=\left|S^{n-1}\right| e^{I(0)} .
$$


2. $I(|x|) \rightarrow-\infty$ as $x \rightarrow O$.

We see that

$$
Z(x)=o\left(|x|^{2-n}\right) \quad \text { as } x \rightarrow O,
$$

and we can solve (4.37) to find $C=0$. Thus, in this case we obtain the interesting corollary that

$$
\mathcal{L} u=0 \text { in } B_{\varepsilon}
$$

admits a solution $u=Z$ that is quite singular at $x=O$ :

$$
|Z(x)| \geq C_{\lambda}|x|^{2-n+\lambda}
$$

for every $\lambda>0$. In particular, local regularity of solutions of the homogeneous equation (4.39) does not hold.

3. $I(|x|) \rightarrow \infty$ as $x \rightarrow O$.

Now we find that

$$
Z(x)|x|^{n-2} \rightarrow \infty \text { as } x \rightarrow O,
$$

so this solution grows more rapidly than the fundamental solution for the Laplacian. Although $Z$ still satisfies (4.37), we can no longer find $C$.

Acknowledgments We express our deep gratitude to a referee for a number of valuable suggestions which helped us to improve the exposition.

Open Access This article is distributed under the terms of the Creative Commons Attribution License which permits any use, distribution and reproduction in any medium, provided the original author(s) and source are credited.

\section{References}

1. Adams, D.R., Hedberg, L.-I.: Function Spaces and Potential Theory. Springer, Berlin (1996)

2. Adolfsson, V., Pipher, J.: The inhomogeneous Dirichlet problem for $\Delta^{2}$ in Lipschitz domains. J. Funct. Anal. 159(1), 137-190 (1998)

3. Agranovich, M.S.: To the theory of the Dirichlet and Neumann problems for strongly elliptic systems in Lipschitz domains. Funct. Anal. Appl. 41(4), 247-263 (2007)

4. Agranovich, M.S.: Remarks on potential spaces and Besov spaces in a Lipschitz domain and on Whitney arrays on its boundary. Russ. J. Math. Phys. 15(2), 146-155 (2008)

5. Amrouche, C., Girault, V.: On the existence and regularity of the solution of Stokes problem in arbitrary dimension. Proc. Japan Acad. Ser. A Math. Sci. 67(5), 171-175 (1991)

6. Auscher, P., Axelsson, A., McIntosh, A.: On a quadratic estimate related to the Kato conjecture and boundary value problems. Contemp. Math. 205, 105-129 (2010)

7. Auscher, P., Axelsson, A., McIntosh, A.: Solvability of elliptic systems with square integrable boundary data. Ark. Mat. 48(2), 252-287 (2010)

8. Auscher, P., Hoffman, S., Lacey, M., McIntosh, A., Tchamitchian, P.: The solution of the Kato square root problem for second order elliptic equations in $\mathbf{R}^{n}$. Ann. Math. 152(2), 633-654 (2002) 
9. Auscher, P., Qafsaoui, M.: Observations on estimates for divergence elliptic equations with VMO coefficients. Boll. Unione Mat. Ital. Sez. B Artic. Ric. Mat. 5, 487-509 (2002)

10. Buffa, A., Geymonat, G.: On traces of functions in $W^{2, p}(\Omega)$ for Lipschitz domains in $\mathbb{R}^{3}$. C. R. Acad. Sci. Paris Sér. I Math. 332(8), 699-704 (2001)

11. Burago, Yu.D., Maz'ya, V.G.: Some questions of potential theory and function theory for domains with non-regular boundaries, Zap. Nauchn. Semin. Leningr. Otd. Mat. Inst. Steklova, vol. 3, pp. 1-152 (Russian) (1967). English translation: Seminars in Math., V. A. Steklov Math, Inst., Leningrad, vol. 3, pp. 1-68. Consultants Bureau, New York (1969)

12. Caffarelli, L.A., Peral, I.: On $W^{1, p}$ estimates for elliptic equations in divergence form. Commun. Pure Appl. Math. 51(1), 1-21 (1998)

13. Cattabriga, L.: Su un problema al contorno relativo al sistema di equazioni di Stokes. Rend. Sem. Mat. Univ. Padova 31, 308-340 (1961)

14. Cianchi, A., Maz'ya, V.: Global Lipschitz regularity for a class of quasilinear elliptic equations. Commun. Partial Diff. Equ. 36(1), 103-133 (2011)

15. Chiarenza, F., Frasca, M., Longo, P.: Solvability of the Dirichlet problem for nondivergence elliptic equations with $V M O$ coefficients. Trans. Am. Math. Soc. 336, 841-853 (1993)

16. Dahlberg, B.E.: Poisson semigroups and singular integrals. Proc. Am. Math. Soc. 97, 41-48 (1986)

17. Dahlberg, B.E.: On the absolute continuity of elliptic measures. Am. J. Math. 108(5), 1119-1138 (1986)

18. Dahlberg, B.E., Kenig, C.E.: Hardy spaces and the Neumann problem in $L^{p}$ for Laplace's equation in Lipschitz domains. Ann. Math. 125(3), 437-465 (1987)

19. Dahlberg, B.E., Kenig, C.E., Pipher, J., Verchota, G.C.: Area integral estimates for higher order elliptic equations and systems. Ann. Inst. Fourier (Grenoble) 47(5), 1425-1461 (1997)

20. Dautray, R., Lions, J.-L.: Mathematical Analysis and Numerical Methods for Science and Technology, vol. 6. Springer, Berlin (1993)

21. De Giorgi, E.: Nuovi teoremi relative alle misure $(r-1)$-dimensionale in spazio ad $r$ dimensioni. Ric. Mat. 4, 95-113 (1955)

22. De Giorgi, E.: Un esempio di estremali discontinue per un problema variazionale di tipo ellittico. Boll. Un. Mat. Ital. 1(4), 135-137 (1968)

23. Di Fazio, G.: $L^{p}$ estimates for divergence form elliptic equations with discontinuous coefficients. Boll. Un. Mat. Ital. A (7) 10(2), 409-420 (1996)

24. Dindoš, M., Kenig, C., Pipher, J.: BMO solvability and the $A_{\infty}$ condition for elliptic operators. arXiv: 1007.5496v1 [math. AP]

25. Dindoš, M., Maz’ya, V.: $L^{p}$ solvability of the stationary Stokes problem in domains with conical singularity in any dimansion. J. Math. Sci. 170(1), 1-19 (2010)

26. Dindoš, M., Mitrea, M.: The stationary Navier-Stokes system in nonsmooth manifolds: the Poisson problem in Lipschitz and $C^{1}$ domains. Arch. Ration. Mech. Anal. 174(1), 1-47 (2004)

27. Fabes, E., Jerison, D., Kenig, C.: Necessary and sufficient conditions for absolute continuity of elliptic-harmonic measure. Anal. Math. 119(2), 121-141 (1984)

28. Federer, H.: A note on the Gauss-Green theorem. Proc. Am. Math. Soc. 9, 447-451 (1958)

29. Gagliardo, E.: Caratterizzazioni delle trace sulla frontiera relative ad alcune classi di funzioni in piu variabili. Rend. Sem. Mat. Univ. Padova 27, 284-305 (1957)

30. Galdi, G.P., Simader, C.G., Sohr, H.: On the Stokes problem in Lipschitz domains. Ann. Math. Pura Appl. 167(4), 147-163 (1994)

31. Giga, Y.: Analyticity of the semigroup generated by the Stokes operator in $L^{r}$ spaces. Math. Z. 178(3), 297-329 (1981)

32. Guidetti, D.: General linear boundary value problems for elliptic operators with VMO coefficients. Math. Nachr. 237, 62-88 (2002)

33. Hofmann, S., Lewis, J.L.: $L^{2}$ solvability and representation by caloric layer potentials in time-varying domains. Ann. Math. 144(2), 349-420 (1996)

34. Hofmann, S., Mitrea, M., Taylor, M.: Geometric and transformational properties of Lipschitz domains, Semmes-Kenig-Toro domains, and other classes of finite perimeter domains. J. Geom. Anal. 17(4), 593-647 (2007)

35. Hörmander, L.: The Analysis of Linear Partial Differential Operators, vol. 1. Springer, Berlin (1983)

36. Jerison, D., Kenig, C.: The inhomogeneous Dirichlet problem in Lipschitz domains. J. Funct. Anal. 130, 161-219 (1995)

37. John, F.: Plane Waves and Spherical Means Applied to Partial Differential Equations. Interscience, New York (1955) 
38. Jonsson, A., Wallin, H.: Function Spaces on Subsets of $\mathbb{R}^{n}$, University of Umea (1984)

39. Kenig, C.: Harmonic Analysis Techniques for Second Order Elliptic Boundary Value Problems, Regional Conference Series in Mathematics, vol. 83, AMS, Providence (1994)

40. Kondrat'iev, V.A.: Boundary value problems for elliptic equations in domains with conical and angular points, Trudy Moscov. Mat. Obshch. 16, 209-292 (1967). English transl.: Trans. Moscow Math. Soc. 16, 227-313 (1967)

41. Kozlov, V.A., Mazya, V.: Differential Equations with Operator Coefficients. Springer, Berlin (1999)

42. Kozlov, V.A., Mazya, V.: Asymptotic formula for solutions to the Dirichlet problem for elliptic equations with discontinuous coefficients near the boundary. Ann. Scuola. Norm. Sup. Pisa 2(3), 551600 (2003)

43. Kozlov, V.A., Mazya V.: Asymptotics of a singular solution to the dirichlet problem for elliptic equations with discontinuous coefficients near the boundary. In: Function Spaces, Differential Operators and Nonlinear Analysis, pp. 75-115. Birkhäuser, Basel (2003)

44. Kozlov, V.A., Maz'ya, V.: Asymptotic formula for solutions to elliptic equations near Lipschitz boundary. Ann. Mat. Pura Appl. 184, 185-213 (2005)

45. Kozlov, V.A., Mazya, V., Rossmann, J.: Spectral Problems Associated with Corner Singularities of Solutions to Elliptic Equations, Mathematical Surveys and Monographs, vol. 85. American Mathematical Society, Providence (2000)

46. Maz'ya, V.: Examples of nonregular solutions of quasilinear elliptic equations with analytic coefficients. Funct. Anal. Appl. 2, 230-234 (1968)

47. Maz'ya, V.: Sobolev Spaces. Springer, Berlin (1985)

48. Maz'ya, V.: Boundedness of the gradient of a solution to the Neumann-Laplace problem in a convex domain. C.R. Acad. Sci. Paris, Ser. I 347, 517-520 (2009)

49. Maz'ya, V., McOwen, R.: Asymptotics for solutions of elliptic equations in double divergence form. Commun. Partial Differ. Equ. 32, 1-17 (2007)

50. Maz'ya, V., McOwen, R.: On the fundamental solution of an elliptic equation in nondivergence form. In: Nonlinear Partial Differential Equations and Related Topics. American Mathematical Society, Providence (2010)

51. Maz'ya, V., Mitrea, M., Shaposhnikova, T.: The Dirichlet problem in Lipschitz domains with boundary data in Besov spaces for higher order elliptic systems with rough coefficients. arXiv: math AP/0701898v1, 31 Jan 2007. J. Anal. Math. 110, 167-239 (2010)

52. Maz'ya, V., Mitrea, M., Shaposhnikova, T.: The inhomogeneous Dirichlet problem for the Stokes system in Lipschitz domains with unit normal close to VMO. J. Funct. Anal. Appl. 43(3), 217-235 (2009)

53. Maz'ya, V., Rossmann, J.: Mixed boundary value problems for the Navier-Stokes system in polyhedral domains. Arch. Rational Mech. Anal. 194(2), 669-712 (2009)

54. Maz'ya, V., Rossmann, J.: Elliptic equations in polyhedral domains. In: Mathematical Surveys and Monographs, vol. 162. American Mathematical Society, Providence (2010)

55. Maz'ya, V., Shaposhnikova, T.: On the regularity of the boundary in $L_{p}$-theory of elliptic boundary value problems, Part I: Trudy Sem. S.L. Soboleva, Novosibirsk, vol. 2, pp. 39-56 (1980); Part II: Trudy Sem. S.L. Soboleva, Novosibirsk, vol. 1, pp. 57-102 (1981)

56. Maz'ya, V., Shaposhnikova, T.: Theory of Multipliers in Spaces of Differentiable Functions, Monographs and Studies in Mathematics vol. 23. Pitman, Boston (1985)

57. Maz'ya, V., Shaposhnikova, T.: Theory of Sobolev Multipliers with Applications to Differential and Integral Operators, Grundlehren der Mathematischen Wissenschaften, vol. 337. Springer, Berlin (2009)

58. Meyers, N.G.: An estimate for the gradient of solutions of second order elliptic divergence equations. Ann. Scuola Norm. Sup. Pisa 17(3), 189-206 (1963)

59. Mitrea, D., Mitrea, M., Taylor, M.: Layer potentials, the Hodge Laplacian, and global boundary problems in nonsmooth Riemannian manifolds. Memoirs of the American Mathematical Society, Provedence, vol. 150, no. 713 (2001)

60. Mitrea, M., Taylor, M.: Navier-Stokes equations on Lipschitz domains in Riemannian manifolds. Math. Ann. 321(4), 955-987 (2001)

61. Nečas, J.: Les Méthodes Directes en Théorie des Équations Elliptiques, Masson et Cie, Éditeurs, Paris, Academia, Éditeurs, Prague (1967)

62. Palagachev, D.K., Softova, L.G.: Singular integral operators, Morrey spaces and fine regularity of solutions to PDE's. Potential Anal. 20, 237-263 (2004)

63. Pipher, J., Verchota, G.C.: Dilation invariant estimates and the boundary Gårding inequality for higher order elliptic operators. Ann. Math. 142(1), 1-38 (1995) 
64. Runst, T., Sickel, W.: Sobolev spaces of fractional order, Nemytskij operators, and nonlinear partial differential equations. Walter de Gruyter, Berlin (1996)

65. Rychkov, V.: On restrictions and extensions of the Besov and Triebel-Lizorkin spaces with respect to Lipschitz domains. J. Lond. Math. Soc. (2) 60(1), 237-257 (1999)

66. Shen, Z.: A note on the Dirichlet problem fir the Stikes system in Lipschitz domains. Proc. Am. Math. Soc. 123, 801-811 (1995)

67. Solonnikov, V.A.: General boundary value problems for systems elliptic in the sense of A. Douglis and L. Nirenberg, II. Trudy Mat. Inst. Steklov 92, 233-297 (1966)

68. Stroffolini, B.: Elliptic systems of PDE with BMO-coefficients. Potential Anal. 15, 285-299 (2001)

69. Temam, R.: Navier-Stokes Equations. Theory and Numerical Analysis, Studies in Mathematics and its Applications, vol. 2. North-Holland Publishing Co., Amsterdam (1977)

70. Uspenskiĭ, S.V.: Imbedding theorems for classes with weights. Trudy Mat. Inst. Steklov. 60, 282-303 (1961)

71. Verchota, G.C.: The biharmonic Neumann problem in Lipschitz domains. Acta Math. 194, 217-279 (2005)

72. Whitney, H.: Analytic extensions of differentiable functions defined in closed sets. Trans. Am. Math. Soc. 36, 63-89 (1934) 